\title{
IEEE 802.11 Wireless LAN Security Performance Using Multiple Clients
}

Honours Project Report, 2003

Nilufar Baghaei nab49@student.canterbury.ac.nz Department of Computer Science and Software Engineering University of Canterbury, Christchurch, New Zealand

Supervisor: Assoc. Prof. Ray Hunt 


\begin{abstract}
IEEE 802.11 Wireless Networks have gained popularity, providing users mobility and flexibility in accessing information. Existing solutions for wireless LAN networks have been exposed to security vulnerabilities. Previous study has evaluated the security performance of IEEE 802.11 wireless networks using single server-client architecture. This research investigated the effect of multiple security mechanisms on the performance of multi-client congested and un-congested networks. The effect of different TCP and UDP packet sizes on performance of secure networks was also studied. The results showed that WEP encryption significantly degrades the performance of congested wireless networks. Network performance degradation increased as the number of clients was increased under all security mechanisms.
\end{abstract}

\title{
Acknowledgements
}

I would like to thank my supervisor, Associate Professor Ray Hunt, for his support and guidance throughout the year, Aun Haider for our earlier discussion on congestion, Associate Professor Krzysztof Pawlikowski for his helpful comments and Jenne Wong for Windows operating system directions. I also like to thank Dr. Malcolm Shore and Craig Miller for discussing the results with me, and Lucent Technologies and MediaLab for providing the wireless equipment. Thanks also to my family for the support they have given me, and to the 2003 fourth year students, who made this year enjoyable. 


\section{Contents}

1 Introduction 4

2 Background $\quad 5$

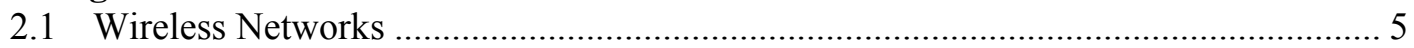

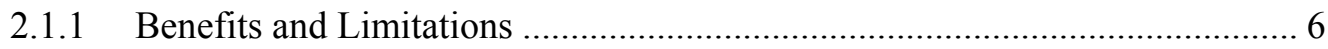

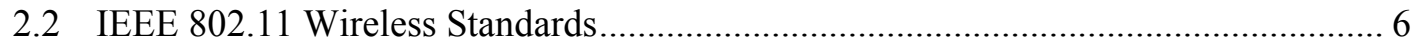

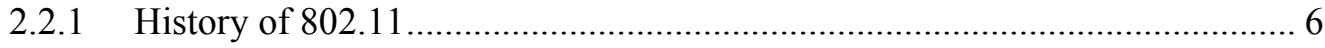

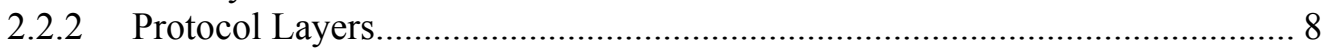

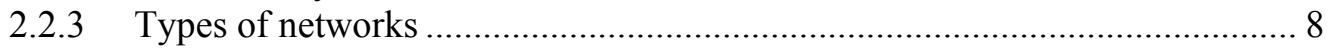

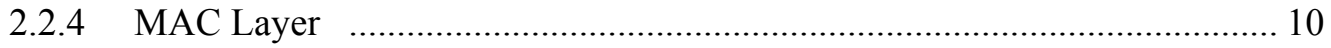

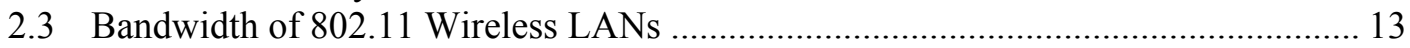

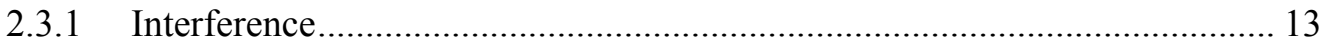

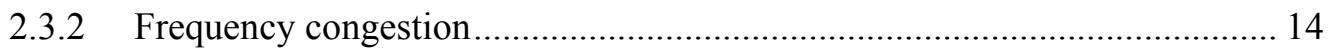

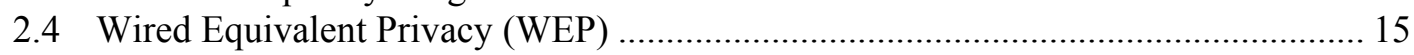

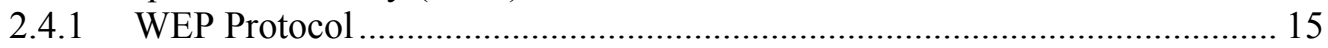

2.4.2 Problems with WEP _............................................................. 16

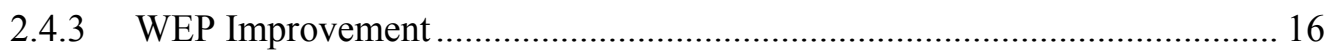

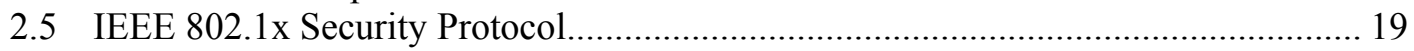

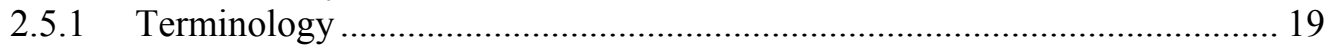

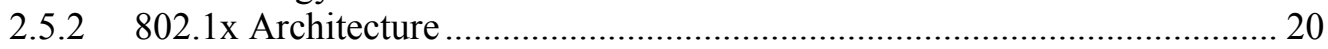

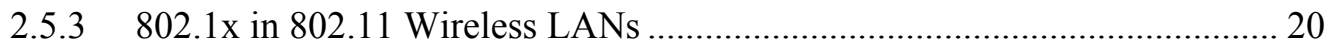

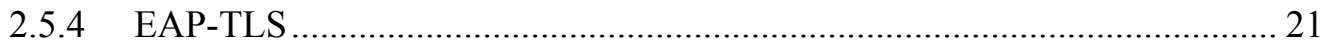

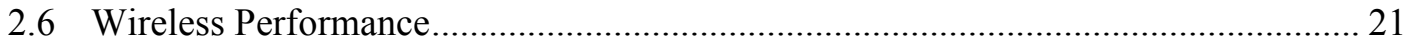

3 Experimets $\quad 24$

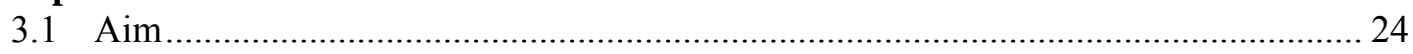

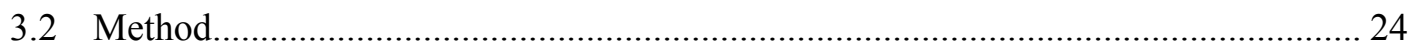

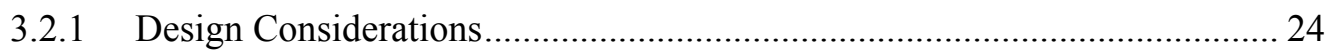

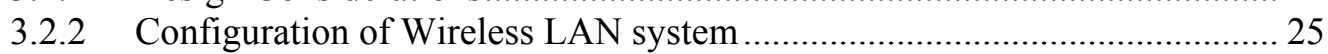

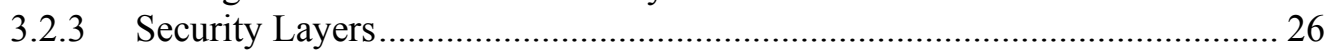

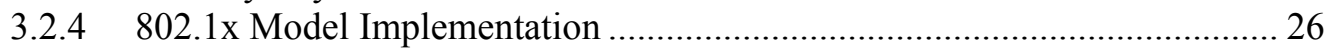

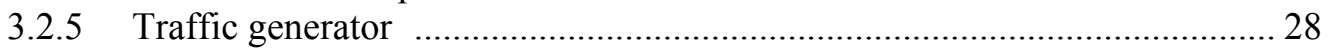

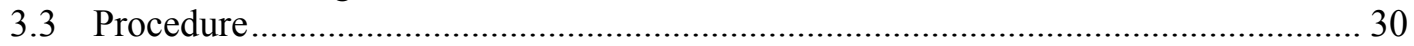

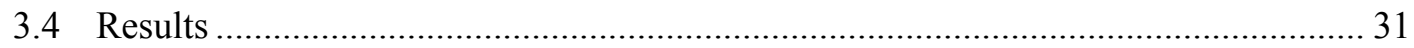

3.4.1 Effect of security mechanisms on performance ……................................. 31

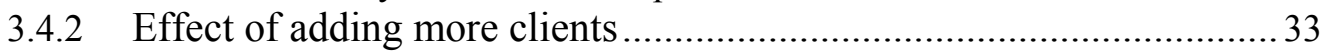

3.4.3 Effect of various packet sizes on performance............................................ 34

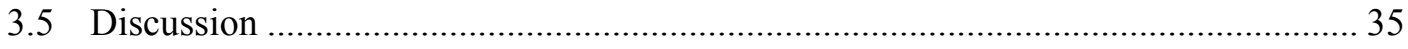

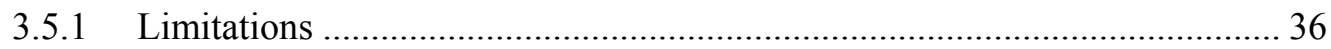

$\begin{array}{ll}4 \text { Future Work } & 37\end{array}$

5 Conclusions $\quad 38$

$\begin{array}{ll}\text { Appendix A } & 44\end{array}$

$\begin{array}{lr}\text { Appendix B } & 45\end{array}$ 


\section{Chapter 1}

\section{Introduction}

The market for wireless communications has experienced incredible growth over recent years. Wireless Local Area Networks (Wireless LANs) have quickly found a significant place and popularity in business and the computer industry alike [17]. The major benefit of wireless LANs is increased flexibility and mobility [12]. Unlike a traditional wired LAN, which requires a wire to connect a computer to the network, users can access wireless LANs from nearly anywhere without any restriction; that has greatly increased wireless LANs' popularity. This significant reliance on wireless networks makes it tremendously important to maintain reliable and secure links between the communicating parties.

Security risks in wireless networks are equal to the sum of the risk of operating a wired network plus the new risks introduced due to the portability of wireless devices [3]. To reduce these risks, organizations need to adopt security measures and practices that help bring down their risks to a manageable level.

This research will investigate the performance and security issues of an IEEE 802.11 wireless LAN with the layered security model, using multiple clients. It will study the interaction between different security layers and their effects on performance (response time and throughput) of congested and un-congested networks. The research will also evaluate the effect of different TCP and UDP packet sizes on network performance, under different security mechanisms.

Chapter 2 provides an introduction to existing wireless networks and detailed analysis of IEEE 802.11 protocols and standards is presented. It explains the two security architectures: WEP (an IEEE 802.11 security standard) and IEEE 802.1x authentication protocol (an enhancement for default WEP authentication). It also discusses the prior research carried out to evaluate the performance of wireless networks. Chapter 3 presents the goal of this research, the design considerations and the different security mechanisms used during the experiments. The model and system architecture is described and the experimented results are presented and analysed. Direction for future work is presented in Chapter 4. Chapter 5 draws some conclusion on the research presented in this report. 


\section{Chapter 2}

\section{Background}

\subsection{Wireless Networks}

Over recent years, the market for wireless communications has experienced incredible growth. Wireless technologies have quickly found a significant place and popularity in business and the computer industry. Their major motivation and benefit is increased flexibility and mobility. Unlike a traditional wired network, which requires a wire to connect a computer to the network, wireless technology enables the users to access information from anywhere without any restriction. Wireless networks are frequently categorised into three groups based on their coverage range [3]: Wireless Wide Area Network (WWAN), Wireless Local Area Network (WLAN), and Wireless Personal Area Network (WPAN).

Wireless WANs include wide coverage area technologies such as Advanced Mobile Phone Systems (AMPS), Time Division Multiple Access (TDMA), and the Code Division Multiple Access (CDMA). Existing second-generation (2G) digital cellular systems are Global System for Mobile (GSM) in Europe, and Personal Digital Communication (PDC) in Japan. The 2G to $2.5 \mathrm{G}$ wireless WANs provides data rate from $9.6 \mathrm{Kbps}$ to $348 \mathrm{Kbps}$. As for thirdgeneration (3G) systems, Universal Mobile Telecommunication System (UMTS) is one of the major systems aiming for higher capacity and data rates with global mobility, and operates around $144 \mathrm{Kbps}$ to $2 \mathrm{Mbps}$ [8].

Wireless LANs provide greater flexibility and portability than do traditional wired LANs. Unlike a wired LAN, which requires a wire to access the network, a Wireless LAN connects computers and other components to the network via an Access Point (AP). IEEE 802.11 is an international standard providing transmission speeds ranging from $1 \mathrm{Mbps}$ to $54 \mathrm{Mbps}$ in either the $2.4 \mathrm{GHz}$ or $5 \mathrm{GHz}$ frequency bands. Section 2.2 discusses the 802.11 standard in more detail. High performance radio $L A N^{1}$ is another wireless LAN standard operating in the $5 \mathrm{GHz}$ frequency band. HiperLAN/1 has a transmission speed of $19 \mathrm{Mbps}$, while HiperLAN/2 operates at 54 Mbps. HiperLAN/2 supports Quality of Service (QoS) and is based on an infrastructure topology.

Wireless PANs typically provide a maximum range of 10 meters, facilitating communication between laptops, cell phones and Personal Digital Assistants (PDAs). The best-known Wireless PAN technology, Bluetooth ${ }^{2}$, is based on low power signaling in the $2.4 \mathrm{GHz}$ frequencies similar to the $802.11 \mathrm{~b}$ standard, but using a different approach to signal processing. It is intended to provide wireless links between mobile computers, PDA's, cell phones and the Internet. Significant operational differences between Bluetooth and 802.11b are the bandwidth, $1 \mathrm{Mbps}$ versus $11 \mathrm{Mbps}$, and distance, $10 \mathrm{M}$ versus $100 \mathrm{M}$. IEEE 802.15 is another Wireless PAN technology that aims at very low power consumption, and operates at 10 meters with data rates less than 1 Mbps. The 802.15 WPAN standard targets interoperability between Wireless PAN devices and devices meeting the IEEE 802.11 standard [8].

\footnotetext{
${ }^{1}$ http://www.hiperlan2.com

2 http://www.bluetooth.com
} 
Figure 2-1 (adapted from [13]) illustrates the three main categories of wireless networks and their coverage ranges. The most successful wireless networking technology this far has been 802.11 [4] and hence is the main focus of this research.

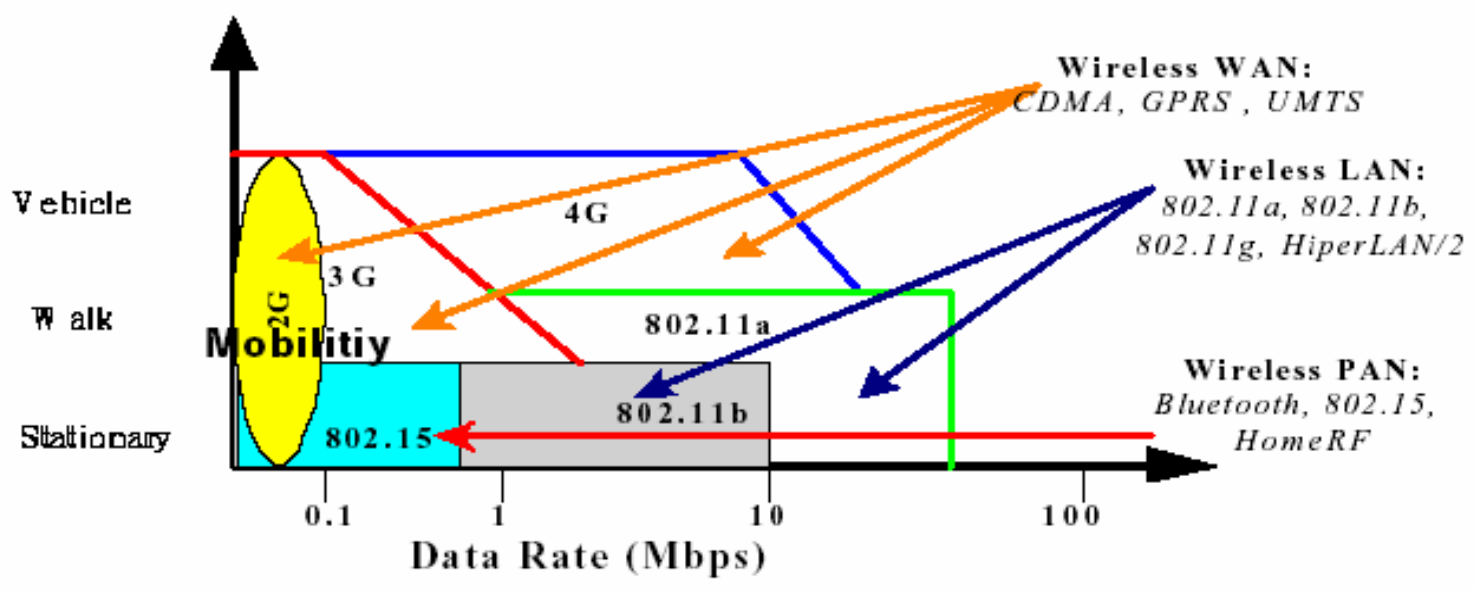

Figure 2-1: Overview of Wireless Networks

\subsubsection{Benefits and Limitations}

Wireless LANs offer several fundamental benefits including user mobility, rapid installation, flexibility and scalability. However, there are some primary limitations [4]:

- The speed of wireless networks is constrained by the available bandwidth. Information Theory can be used to deduce the upper limit on the speed of a network. Wireless network hardware tends to be slower than wired hardware. Unlike the Ethernet standard, wireless standards must carefully validate received frames to guard against loss due to the unreliability of the wireless medium.

- Using radio waves as the network medium poses several challenges. Specifications for wired networks are designed so that a network will work as long as it meets the specifications. Radio waves can suffer from a number of propagation problems that may interrupt the radio link, such as multi-path interference and shadows.

- Security on any network is a prime concern. On wireless networks, it is often a critical concern because the network transmissions are available to anyone within range of the transmitter with the appropriate antenna. On a wired network, the signals stay in the wires and can be protected by strong physical-access control. On a wireless network, sniffing is much easier because the radio transmissions are designed to be processed by any receiver within range.

\subsection{IEEE 802.11 Wireless Standards}

\subsubsection{History of 802.11}

The IEEE breaks their standards into various committees. The IEEE 802 Committee deals with Local and Metropolitan Area Networks. The 802 series of standards is broken into 
working groups that focus on specific issues within the overall discipline of LANs and MANs [9].

The following is a list of some of the 802 working groups:

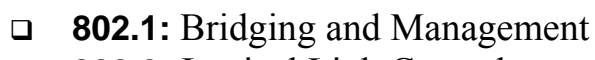

- 802.2: Logical Link Control

a 802.3: $\mathrm{CSMA} / \mathrm{CD}$ Access Method

a 802.4: Token-Passing Bus Access Method

a 802.7: Broadband LAN

a 802.11: Wireless

The 802.11 Working Group was formed in September of 1990. Their goal was to create a wireless LAN specification that will operate in one of the Industrial, Scientific, and Medical (ISM) frequency ranges, The first 802.11 standard was released in 1997 [17].

The 802 standards address the lower levels of the OSI model. The 802.11 protocols address the Medium Access Control (MAC) and Physical (PHY) layers independently. The MAC layer handles moving data between the link layer and the physical medium. Figure 2-2 illustrates how the lower layers of the OSI model match up to the concepts outlined in the 802 series of protocols.

There are many different PHY standards in use nowadays. The original 802.11 specification documented three different mechanisms: Infrared (IR), 2.4 GHz Frequency Hopping Spread Spectrum (FHSS), and $2.4 \mathrm{GHz}$ Direct Sequence Spread Spectrum (DSSS). All these mechanisms provided 1 or 2 Mbps data rate depending on the signal quality.

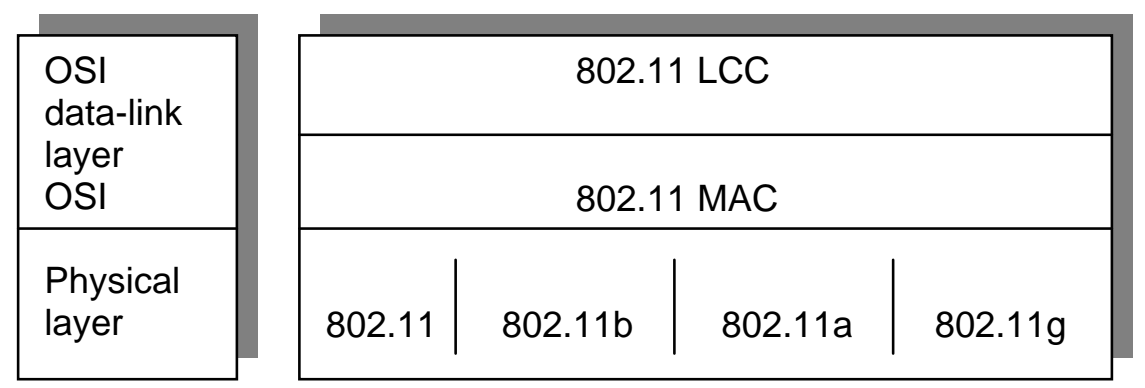

Figure 2-2: The OSI layers and corresponding 802 structure

The specific groups and tasks concerning wireless networking hardware standards are as follows:

$802.11 b$

$802.11 \mathrm{~b}[19,22]$, released on 1999 , specified a new PHY that provided a higher bit rate using DSSS in the $2.4 \mathrm{GHz}$ range. $802.11 \mathrm{~b}$ can transmit data up to $11 \mathrm{Mbps}$ but will scale down to $1 \mathrm{Mbps}$ based on conditions. Due to the higher bit rate and increased interpretability, $802.11 \mathrm{~b}$ has gained rapid deployment. Interoperability between different 802.11 products is tested and certified by Wireless Ethernet Compatibility Alliance $\left(\mathrm{WECA}^{3}\right)$ and their certification mark is $\mathrm{Wi}^{-} \mathrm{Fi}^{4}$.

\footnotetext{
${ }^{3}$ The WECA includes Cisco, 3Com, Enterasys, Lucent, and many other wireless networking companies. For more information, refer to http://www.weca.net/.

${ }^{4}$ Wireless Fidelity
} 


\section{$802.11 \mathrm{a}$}

802.11a [18], released in 2001, operates in the $5 \mathrm{GHz}$ range. It provides a bit rate of up to $54 \mathrm{Mbps}$ and uses a modulation method called Orthogonal Frequency Division Multiplexing (OFDM). Some vendors have proprietary implementations that double the bit rate of 802.11 a to $102 \mathrm{Mbps}$.

\section{일.11g}

$802.11 \mathrm{~g}$ [20] operates in the same $2.4 \mathrm{GHz}$ range as $802.11 \mathrm{~b}$ but uses OFDM similar to $802.11 \mathrm{a}$. Operating at up to $22 \mathrm{Mbps}$, it is seen as the middleman between $802.11 \mathrm{~b}$ and 802.11a standards.

Table 2-1 summarises the 802.11 PHY specifications. $802.11 \mathrm{~b}$ is currently the most deployed type of wireless LAN and is used in the experimentation part of this research.

\begin{tabular}{|c|r|r|r|}
\hline 802.11 PHY & Max Data Rate & \multicolumn{1}{|c|}{ Frequency } & \multicolumn{1}{c|}{ Modulation } \\
\hline 802.11 & $2 \mathrm{Mbps}$ & $2.4 \mathrm{GHz} \& \mathrm{IR}$ & FHSS \& DSSS \\
\hline $802.11 \mathrm{~b}$ & $11 \mathrm{Mbps}$ & $2.4 \mathrm{GHz}$ & DSSS \\
\hline $802.11 \mathrm{~g}$ & $22 \mathrm{Mbps}$ & $2.4 \mathrm{GHZ}$ & OFDM \\
\hline $802.11 \mathrm{a}$ & $54 \mathrm{Mbps}$ & $5 \mathrm{GHZ}$ & OFDM \\
\hline
\end{tabular}

Table 2-1: PHY specifications

\subsubsection{Protocol Layers}

The physical layer defines the frequency band, data rate, and other details of the actual radio transmission. Above the physical layer is the MAC layer that regulates access to the shared radio frequency band so that station transmissions do not interfere with one another. The MAC layer has two sub-layers: the lower one is the distributed coordination function (DCF), which uses an Ethernet-style contention algorithm that provides access to all traffic. Ordinary asynchronous traffic uses this coordination function (see Section 2.2.4). The upper MAC sublayer is the point coordination function (PCF), a centralised MAC algorithm that provides contention-free service by polling stations in turn. Higher priority traffic - traffic with greater timing requirements - uses this coordination function (see Section 2.2.4). Finally, the logical link control layer provides an interface to higher layers and performs basic link layer functions such as error control (Figure 2-3, adapted from [17, 56]).

\subsubsection{Types of networks}

The 802.11 standard defines two modes: infrastructure mode and ad hoc mode. In infrastructure mode (Figure 2-4), the wireless network consists of at least one AP connected to the wired network infrastructure and a set of wireless end stations. This configuration is called a Basic Service Set (BSS). If one mobile station in an infrastructure BSS needs to communicate with a second mobile station, the communication must take two hops. First the originating mobile station transfers the frame to the AP. Second, the AP transfers the frame to the destination station. With all communications relayed through an AP, the basic service area corresponding to an Infrastructure BSS is defined by the points in which transmissions from the AP can be received. 


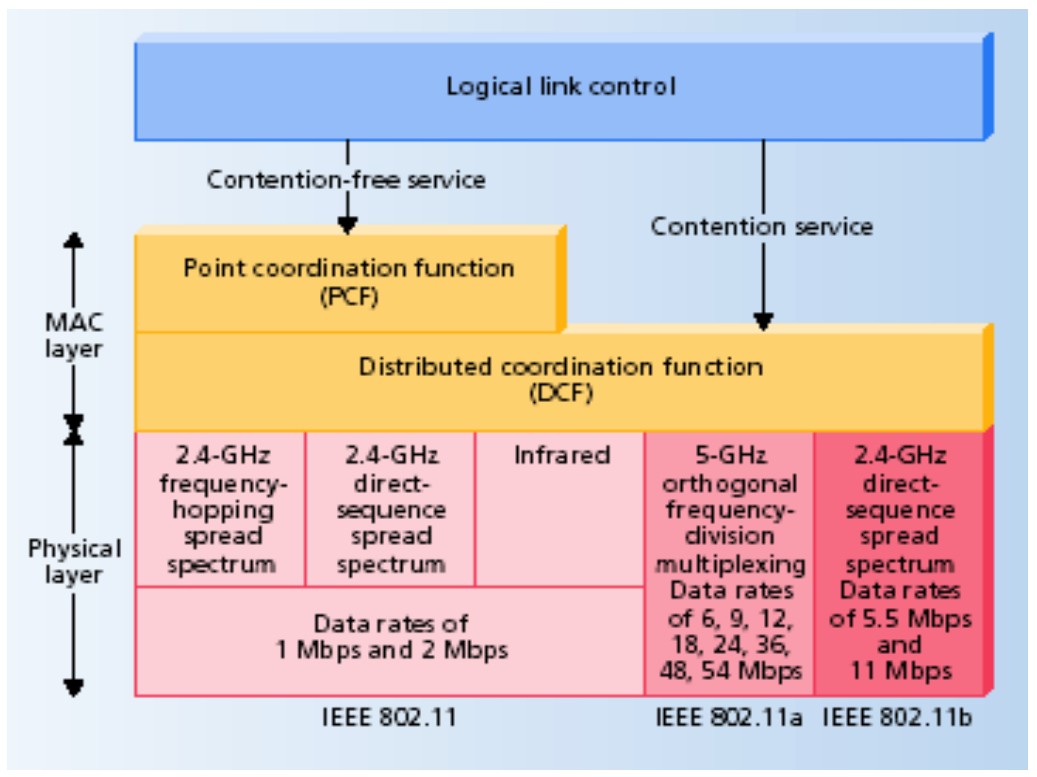

Figure 2-3: The 802.11 Protocol Stack

Although the multi-hop transmission takes more transmission capacity than a directed frame from the sender to the receiver, it has two major advantages [9]:

- An infrastructure BSS is defined by the distance from the AP. All mobile stations are required to be within reach of the AP, but no restriction is placed on the distance between mobile stations themselves. Allowing direct communication between mobile stations would save transmission capacity but at the cost of increased physical layer complexity, since mobile stations would need to maintain neighbour relationships with all other mobile stations within the service area.

a APs in infrastructure networks are in a position to assist with stations attempting to save power. APs can note when a stations enters a power-saving model (see Section 2.2.4) and buffer frames for it. Battery-operated stations can turn the wireless transceiver off and power it up only to transmit and retrieve buffered frames from the AP.

An Extended Service Set (ESS) is a set of two or more BSSs forming a single sub-network. Since most corporate Wireless LANs require access to the wired LAN for services (file servers, printers, Internet links), they will operate in infrastructure mode. This research uses the infrastructure mode for the experiments. 


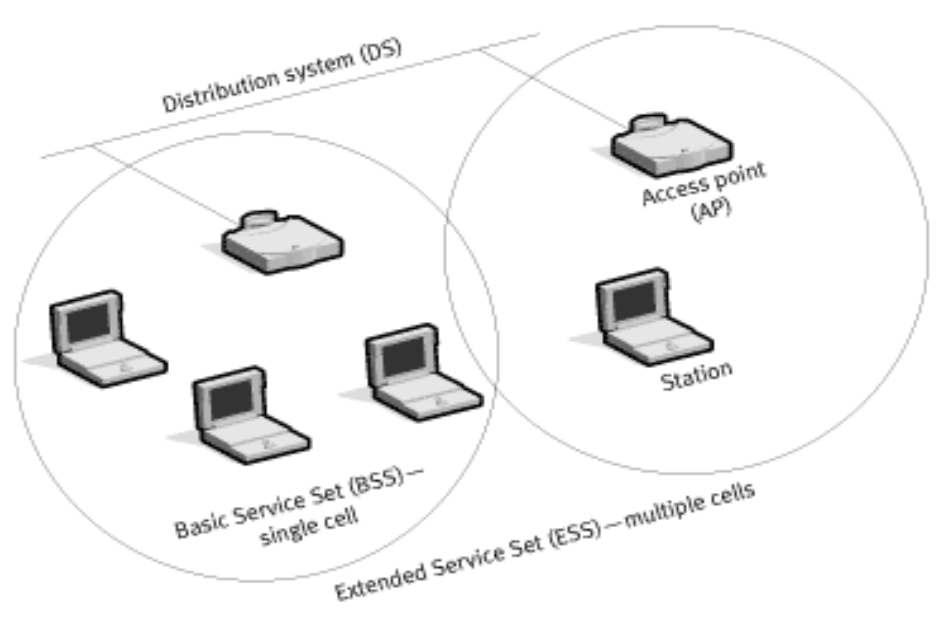

Figure 2-4: Infrastructure mode

Ad hoc mode (also called peer-to-peer mode or an Independent Basic Service Set, or IBSS) is a set of 802.11 wireless stations that communicate directly with one another without using an AP or any connection to a wired network (Figure 2-5). One common use is to create a shortlived network to support a single meeting in a conference room. As the meeting begins, the participants create an IBSS to share data. When the meeting ends, the IBSS is dissolved. Due to their short duration, small size and focused purpose, IBSSs are usually referred to as ad hoc networks.

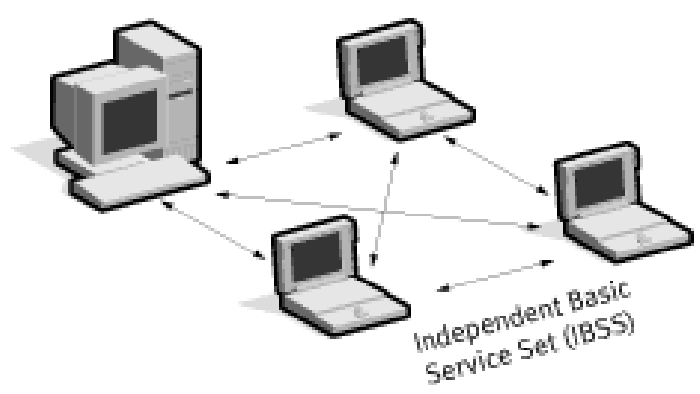

Figure 2-5: Ad hoc mode

\subsubsection{MAC Layer}

Medium Access Control (MAC) is supported by all physical layers. It provides the core framing operations and the interaction with a wired network backbone. Different physical layers may provide different transmission speeds, all of which are supposed to interoperate.

802.11 does not depart from the previous IEEE 802 standards in any major way. The standard successfully adapts Ethernet-style networking to radio links. Similar to Ethernet, 802.11 uses a Carrier Sense Multiple Access (CSMA) scheme to control access to the transmission medium. However, collisions waste valuable transmission capacity, so rather than the Collision Detection (CSMA/CD) employed by Ethernet, 802.11 uses Collision Avoidance (CSMA/CA) [6]. Similar to Ethernet, 802.11 uses a distributed access scheme with no centralised controller. Each 802.11 station uses the same method to gain access to the medium. The major differences between 802.11 and Ethernet come from the differences in the underlying medium. 
The following summarises primary 802.11 MAC functions, especially as they relate to infrastructure wireless LANs $[6,17,59]$ :

- Scanning: The 802.11 standard defines both passive and active scanning; whereby, a radio NIC ${ }^{5}$ searches for APs. Passive scanning is mandatory where each NIC scans individual channels to find the best AP signal. Periodically, APs broadcast a beacon, and the radio NIC receives these beacons while scanning and takes note of the corresponding signal strengths. The beacons ${ }^{6}$ contain information about the AP, including SSID $^{7}$ and supported data rates. The radio NIC can use this information along with the signal strength to compare APs and decide upon which one to use.

Optional active scanning is similar, except the radio NIC initiates the process by broadcasting a probe frame and all APs within range respond with a probe response. Active scanning enables a radio NIC to receive immediate response from APs, without waiting for a beacon transmission; however, this will create additional overhead on the network.

a Authentication: Authentication is the process of proving identity. The 802.11 standard specifies two forms: Open system authentication and shared key authentication. Open system authentication is mandatory, and it is a two-step process. A radio NIC first initiates the process by sending an authentication request frame to the AP. The AP replies with an authentication response frame containing approval or disapproval of authentication indicated in the Status Code field in the frame body.

Shared key authentication is an optional four-step process that bases authentication on whether the authenticating device has the correct WEP (wired equivalent privacy) $\mathrm{key}^{8}$. The radio NIC starts by sending an authentication request frame to the AP. The AP then places challenge text into the frame body of a response frame and sends it to the radio NIC. The radio NIC uses its WEP key to encrypt the challenge text and then sends it back to the AP in another authentication frame. The AP decrypts the challenge text and compares it to the initial text. If they are the same, the AP assumes that the radio NIC has the correct key. The AP finishes the sequence by sending an authentication frame to the radio NIC with the approval or disapproval.

a Association: Once authenticated, the radio NIC must associate with the AP before sending data frames. Association is necessary to synchronise the radio NIC and AP with important information, such as supported data rates. The radio NIC initiates the association by sending an association request frame containing elements such as SSID and supported data rates. The AP responds by sending an association response frame containing an association ID along with other information regarding the AP. Once the radio NIC and AP complete the association process, they can send data frames to each other.

a Privacy: With a wireless LAN, eavesdropping is a major concern because of the ease of capturing a transmission [17]. To assure privacy, IEEE 802.11 provides for the optional use of encryption by specifying a scheme based on the Wired Equivalent Privacy (WEP) algorithm (see Section 2.4). With the optional WEP enabled, the wireless NIC will encrypt the body and not the header of each frame before transmission using a common key, and the receiving station will decrypt the frame

\footnotetext{
5 Network Interface Card.

${ }^{6}$ For more information about beacon frame, refer to [25].

7 Service Set Identifier

${ }^{8}$ For more information, see Section 2.4.
} 
upon receipt using the common key. The 802.11 standard specifies a 40-bit key and no key distribution method, which makes 802.11 wireless LANs vulnerable to eavesdroppers. For stronger protections, some 802.11 vendors offer optional 128-bit encryption. The $802.11 \mathrm{i}$ committee, however, has improved 802.11 security by incorporating 802.1x and stronger encryption into the standard (see Section 2.5).

- RTS/CTS: The optional Request-To-Send and Clear-To-Send (RTS/CTS) function allows the AP to control use of the medium for stations activating RTS/CTS. With most radio NICs, users can set a maximum frame length threshold whereby the radio NIC will activate RTS/CTS. For instance, a frame length of 1,000 bytes will trigger RTS/CTS for all frames larger than 1,000 bytes. The use of RTS/CTS alleviates hidden node problems, that is, where two or more radio NICs cannot hear each other and they are associated with the same AP.

If the radio NIC activates RTS/CTS, it will first send a RTS frame to AP before sending a data frame. The AP will then respond with a CTS frame, indicating that the radio NIC can send the data frame. With the CTS frame, the AP will provide a value in the duration field of the frame header that holds off other stations from transmitting until after the radio NIC initiating the RTS can send its data frame. This avoids collisions between hidden nodes. The RTS/CTS handshake continues for each frame, as long as the frame size exceeds the threshold set in the corresponding radio NIC.

a Power Save Mode: The optional power save mode that a user can turn on or off enables the radio NIC to conserve battery power when there is no need to send data. With power save mode on, the radio NIC indicates its desire to enter sleep state to the AP via a status bit located in the header of each frame. The AP takes note of each radio NIC wishing to enter power save mode, and buffers packets corresponding to the sleeping station. In order to still receive data frames, the sleeping NIC must wake up periodically (at the right time) to receive regular beacon transmissions coming from the AP.

- Fragmentation: The optional fragmentation function enables an 802.11 station to divide data packets into smaller frames. This is done to avoid needing to retransmit large frames in the presence of RF interference (see Section 2.3.1). The bits errors resulting from RF interference are likely to affect a single frame, and it requires less overhead to retransmit a smaller frame rather than a larger one. As with RTS/CTS, users can generally set a maximum frame length threshold whereby the radio NIC will activate fragmentation. If the frame size is larger than the threshold, the radio NIC will break the packet into multiple frames, with each frame no larger than the threshold value.

Access to the wireless medium is controlled by coordination functions. Ethernet-like CSMA/CA access is provided by the distributed coordination function (DCF). If contentionfree service is required, it can be provided by the point coordination function (PCF) that is built on top of the DCF. Contention-free services are provided only in infrastructure networks. The coordination functions are described below ${ }^{9}$ and illustrated in Figure 2-3.

- DCF: The DCF is the basis of the standard CSMA/CA access mechanism. Similar to Ethernet, it first checks to see whether the radio link is clear before transmitting. To avoid collisions, stations use a random backoff after each frame, with the first transmitter seizing the channel. In some circumstances, the DCF may use the

\footnotetext{
${ }^{9}$ For more information, refer to $[6,23]$.
} 
CTS/RTS clearing technique (mentioned above) to further reduce the possibility of collisions.

a PCF: Point coordination provides contention-free services. Special stations called point coordinators are used to ensure that the medium is provided without contention. Point coordinators reside in APs, so the PCF is restricted to infrastructure networks. To gain priority over standard contention-based services, the PCF allows a station to transmit frames after a shorter interval. The PCF is not implemented in the market yet [6].

\subsection{Bandwidth of 802.11 Wireless LANs}

The $802.11 \mathrm{~b}$ standard is generally understood as an $11 \mathrm{Mbps}$ Ethernet LAN running in the 2.4 $\mathrm{GHz}$ ISM radio band. Because of the demands of the protocol, and the multiple factors influencing radio signals, it is very unlikely that the users will ever achieve $11 \mathrm{Mbps}$ as an operational bandwidth on their LANs. The theoretical throughput can be attained by using DCF (see Section 2.2.4) as $75 \%$ of the nominal bit rate [2], although a target of $65 \%$ is commonly observed. Applying this formula to an $11 \mathrm{Mbps} 802.11 \mathrm{~b}$ network, this yields a practical throughput in the range of 6 to $8 \mathrm{Mbps}$. A comparison test was carried out on the 802.11a and 802.11b throughput limits [31]; the author observed the limit for 802.11a was $30.34 \mathrm{Mbps}$ and $6.44 \mathrm{Mbps}$ for $802.11 \mathrm{~b}$. The maximum overall throughput of an $802.11 \mathrm{~b}$ Wireless LAN in a similar study [40] was reported to be about $6.45 \mathrm{Mbps}$ with a standard deviation of $0.02 \mathrm{Mbps}$ for a single station. Another study [57] analysed IEEE 802.11 operation under various assumptions such as time-independent modeling, geometrically distributed packet sizes, etc. Those results also showed that the IEEE 802.11 standard operates at rates lower than a theoretically possible 7.27 Mbps.

The actual amount of bandwidth is largely dependent on interference and frequency congestion, as discussed in the next following sections:

\subsubsection{Interference}

Reduced to its simplest form, wireless networking is a network where the physical wires have been replaced by radio signals. Unlike wires, radio signals are susceptible to a wide variety of physical and radio frequency (RF) interference. This interference will normally manifest itself as a reduction in performance, and occasionally will result in a complete shutdown.

We assume a signal that reaches from point "A", the wireless AP, to point "B", the wireless network interface card in a workstation. The signal might be affected by the following factors $[22,30]$ :

a Distance (a physical factor): The closer the wireless device is to the wireless AP, the stronger the signal, and the better the performance. A stronger signal requires fewer retransmissions. This is usually only a problem at the edges of the range, but is a serious consideration for the designer.

- Physical barriers such as walls and widows: These are obvious sources of interference, but another significant source of interference can result from the placement of furniture and other objects in the space between points " $A$ " and "B" as well as the walls that define the space. This kind of interference is called multi-path propagation. Assuming a line of sight path between points " $A$ " and "B", a portion of the signal will go directly between the two antennae. Another portion of the signal 
will bounce off adjacent walls and furniture, and will arrive at the receiving antenna some time after the original signal. If the delay is sufficient, the receiving station will not be able to decode the signal and will not acknowledge the packet, requiring a retransmission by the sender. This type of interference is most prevalent in a large space with many reflective surfaces such as a warehouse or manufacturing plant, however, is certainly not limited to those types of spaces.

a Radio frequency (RF) interference: RF interference involves the presence of unwanted, interfering RF signals that disrupt normal system operations. The $802.11 \mathrm{~b}$ sets the standard for wireless networking in the $2.4 \mathrm{GHz}$ radio spectrum. This spectrum is known as the ISM (Industrial, Scientific and Medical). It means that there are also other devices that can radiate radio signals on the same frequency as $802.11 \mathrm{~b}$ networking devices. These will include $2.4 \mathrm{GHz}$ wireless telephones, commercial microwave ovens and Bluetooth devices [30]. Additionally, other 802.11b networks in close proximity can interfere with a network. As mentioned before in Section 2.2.4, 802.11 devices use CSMA/CA technique when transmitting packets. This means that they will only transmit when no other device is transmitting. If a device sees another signal, it will wait for that signal to end before attempting to transmit its packet. If there is another signal of sufficient strength on the $2.4 \mathrm{GHz}$ band, an 802.11 device may see it as another 802.11 device and delay transmission. In addition, since these other radiation sources are not necessarily participating in the protocol, they can start at any time and interfere with a packet being transmitted. This will result in a corrupted packet, a subsequent retransmission and hence a poor performance. If the source of the interference is strong enough and continuous, it can completely shut down the network.

Geier [30] suggests some actions to be taken in order to avoid RF interference. These will include analysing the potential for RF interference, preventing them from operating, providing adequate wireless LAN coverage and setting configuration parameters (such as frequency channel) properly.

\subsubsection{Frequency congestion}

$802.11 \mathrm{~b}$ devices share the $2.4 \mathrm{GHz}$ frequency spectrum with a bewildering number of other radiation sources. The spectrum was originally designated as experimental and its primary usage was granted to amateur radio. ISM and RF devices were allowed to use the band under specific rules governing power output and non-interference. RF devices such as $802.11 \mathrm{~b}$, which operate at low power levels, must accept interference from other sources, and may not interfere with the primary user. As the number of $802.11 \mathrm{~b}$ implementations increases, so will the opportunities for interference with other users.

There has already been some studies aiming at developing signaling strategies to minimise interference between $802.11 \mathrm{~b}$ and other RF devices in the band [22], but there are some theoretical limits. The current $802.11 \mathrm{~b}$ standard allows three channels to operate simultaneously within the spectrum. In a multi-tenant building, this may be inadequate for the demand.

In addition to the existing RF devices that can cause interference, RF Lighting is a new technology that could have a serious impact on frequency congestion. In RF Lighting, a bulb containing a mixture of argon and sulphur is exposed to a high frequency RF signal causing it to fluoresce brightly. This technology promises low energy, high output, and long life. It also operates in the $2.4 \mathrm{GHz}$ band, and may promise additional challenges for $802.11 \mathrm{~b}$ users ${ }^{10}$.

\footnotetext{
${ }^{10}$ For more information, see http://wifinetnews.com/archives/001258.html.
} 
A solution to frequency congestion occurrence in the $802.11 \mathrm{~b}$ band is to migrate to the $802.11 \mathrm{a}$ band [22]. This will solve the congestion problem, but at a significant cost since it will require replacement of both APs and wireless network interface cards, and may force some re-design because of smaller distance limitations.

Since 802.11 wireless networks use a shared medium, the more devices that are trying to access it, the lower the effective throughput will be. This is similar to standard wired Ethernet. When an 802.11 device is transmitting, no other device in the network may transmit data. If there are multiple devices trying to send large amounts of data, there will be heavy contention for the airwaves. This congestion gets worse as more machines are added or more data is being transmitted [16].

\subsection{Wired Equivalent Privacy (WEP)}

\subsubsection{WEP Protocol}

Interception of radio communications has been a problem for as long as radios have been used to transmit sensitive information. Since radio transmissions travel in unsecured areas, interception of theses radio signals by an attacker is a real threat. In order to protect the data from eavesdroppers, various forms of encryption have been used.

The 802.11 MAC specification describes an encryption protocol called Wired Equivalent Privacy (WEP). The goal of WEP is to make Wireless LAN communication as secure as wired LAN data transmissions. WEP provides two critical pieces to the wireless security architecture: authentication and confidentiality. It uses a shared key mechanism with a symmetric cipher called $\mathrm{RC} 4{ }^{11}$. The key that a client is using for authentication and encryption of the data stream must be the same key that the AP uses. The 802.11 standard specifies a 40-bit key, however most vendors have also implemented a 104-bit key for greater security $^{12} 13$.

Encryption of the data stream provides confidentiality of the data transmitted between two Wireless LAN devices. The encryption mechanism used in WEP is a symmetric cipher; this means that the key, which is used to encrypt the data, is the same key that will decrypt the data. If both wireless LAN devices do not have the same encryption key, the data transfer fails ${ }^{14}$.

As described in Section 2.2.4, when a station wants to associate with an AP, the station must authenticate itself to the AP first. When the association occurs, the station and AP exchange the type of authentication they will accept. If the authentication type is specified as open, there will effectively be no authentication. The AP and station identify themselves to each other and the association is complete. The devices may also select the shared secret authentication mechanism. Station A will send a random number to station B. Station B encrypts the random umber using WEP and sends the result back to station A. Station A decrypts the received packet and verifies the decrypted payload equals to random number it sent to station B. If the numbers match, station A notifies station B that the authentication was successful and the association is formed (see Figure 2-6, adapted from [5]).

\footnotetext{
${ }^{11}$ Rivest Cipher 4

${ }^{12}$ Microsoft Implementation of Windows XP supports 40-bit and 104-bit keys. Both have been used in the experimental part of this research (see Chapter 3 for more details).

${ }^{13}$ It is worthy to note that keys are often based on passwords that are chosen by users; this typically reduces the effective key size.

${ }^{14}$ For more information about WEP encryption, refer to [3, 24].
} 


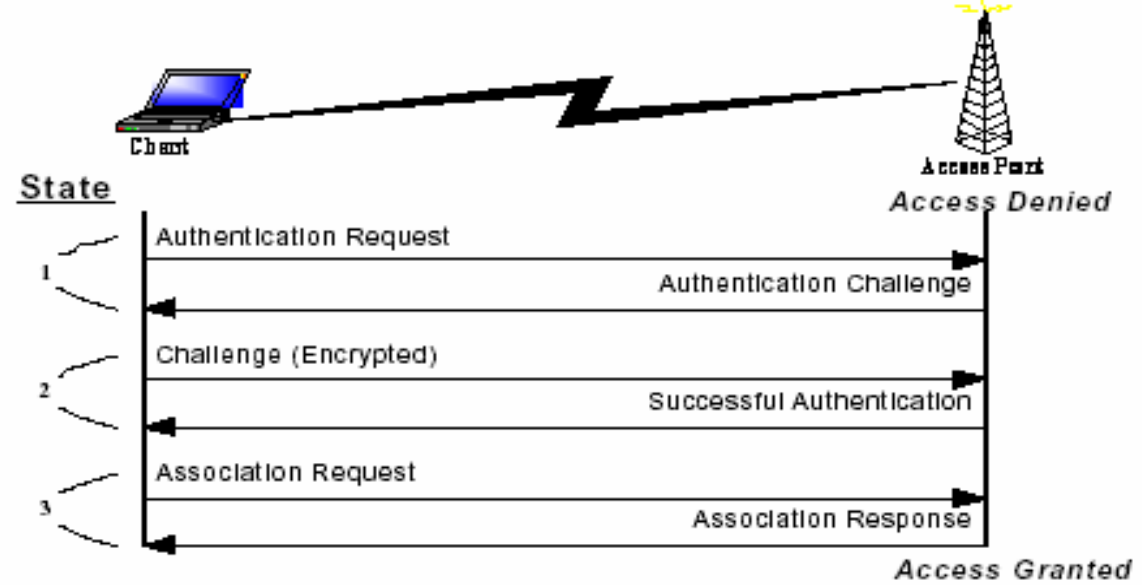

Figure 2-6: Authentication and Association States

\subsubsection{Problems with WEP}

There are some known vulnerabilities in the standardised security of the $802.11 \mathrm{~b}$ Wireless LAN standard. As mentioned above, the WEP protocol is used in 802.11-based Wireless LANs. Several groups of computer security specialists have discovered security problems that let malicious users compromise the security of Wireless LANs. These include passive attacks to decrypt traffic based on statistical analysis, active attacks to inject new traffic from unauthorised mobile stations (i.e., based on known plaintext), active attacks to decrypt traffic (i.e., based on tricking the AP), and dictionary-building attacks [3]. The dictionary building attack is possible after analysing a full day's traffic. Because significant attention is now on the security of 802.11, more attacks are likely to be discovered. Some of the problems associated with WEP and $802.11 \mathrm{~b}$ Wireless LAN security are summarised in Table 2-2 (adapted from [3]).

Fluhrer et al. [15] presented a paper in which the team described a weakness in RC4 as it is implemented in WEP protocol. The issue is not with RC4, but with the way it is used by WEP. The end result is that WEP can be cracked if enough traffic can be intercepted. In addition, as the key length grows, the time it takes grows linearly, while it is supposed to grow exponentially. There are several freely available tools to crack WEP keys, including AirSnort ${ }^{15}$ and WEPCrack ${ }^{16}$. For more information on WEP flaws, refer to [15, 26, 27].

\subsubsection{WEP Improvement}

The link layer security provisions in the 802.11 standards are all vulnerable to attacks. Therefore, systems should deploy additional higher-level security mechanisms such as access control, end-to-end encryption, password protection, authentication, virtual private networks, or firewalls [5] and assume WEP as a very basic layer of security only. The IEEE 802.11 committee has set up task group 802.11i [29] to enhance the security and authentication mechanism of the current 802.11 MAC. Their work has resulted in the development of:

replacement of the 802.11 standard with $802.1 \mathrm{x}[1,32]$ authentication and key management.

\footnotetext{
${ }^{15} \mathrm{http} / / /$ airsnort.shmoo.com

${ }^{16} \mathrm{http://wepcrack.sourceforge.net/}$
} 
- improvement of the exiting WEP with Temporal Key Integrity Protocol (TKIP), also known as WEP2.

- deployment of Enhanced Security Network (ESN) solution with a stronger encryption algorithm ${ }^{17}$.

As described above, one of the major security issues with WEP is the challenge of distributing and managing the encryption keys. The $802.1 \mathrm{x}$ standard has been introduced to provide a centralised authentication and dynamic key distribution for 802.11 architecture using the 802.1x standard with RADIUS [7]. 802.1x - a collaborative effort by vendors in the software, server, and networking industries-is an authentication standard for 802-based LANs using port-based network access control. It is used for communication between wireless clients and APs, while RADIUS operates between an AP and an authentication server. Industry leaders proposed 802.1x to address WEP vulnerabilities by providing access control and key distribution to any (wired or wireless) Ethernet port. The 802.1x standard will be discussed in more details in Section 2.5.

\footnotetext{
${ }^{17}$ For information on WEP2 and ESN, refer to [28, 29].
} 


\section{Security Issue / Vulnerability}

1. Security features in vendor products are frequently not enabled.

2. IVs are short (or static).

3. Cryptographic keys are short.

Cryptographic keys are shared.

5. Cryptographic keys cannot be updated automatically and frequently.

6. RC4 has a weak key schedule and is inappropriately used in WEP.

7. Packet integrity is poor.

\section{Remarks}

Security features, albeit poor in some cases, are not enabled when shipped, and users do not enable when installed. Bad security is generally better than no security.

24-bit IVs cause the generated key stream to repeat. Repetition allows easy decryption of data for a moderately sophisticated adversary.

40-bit keys are inadequate for any system. It is generally accepted that key sizes should be greater than 80 bits in length. The longer the key, the less likely a comprise is possible from a brute-force attack.

Keys that are shared can compromise a system. A fundamental tenant of cryptography is that the security of a system is largely dependent on the secrecy of the keys.

Cryptographic keys should be changed often to prevent bruteforce attacks.

The combination of revealing 24 key bits in the IV and a weakness in the initial few bytes of the RC4 keystream leads to an efficient attack that recovers the key. Most other applications of RC4 do not expose the weaknesses of RC4 because they do not reveal key bits and do not restart the key schedule for every packet. This attack is available to moderately sophisticated adversaries.

CRC32 and other linear block codes are inadequate for providing cryptographic integrity. Message modification is possible. Linear codes are inadequate for the protection against advertent attacks on data integrity. Cryptographic protection is required to prevent deliberate attacks. Use of noncryptographic protocols often facilitates attacks against the cryptography.

8. No user authentication occurs. Only the device is authenticated. A device that is stolen can access the network.

9. Authentication is not enabled; $\quad$ Identity-based systems are highly vulnerable particularly in a only simple SSID identification wireless system. occurs.

10. Device authentication is simple shared-key challenge-response.

One-way challenge-response authentication is subject to "man-inthe-middle" attacks. Mutual authentication is required to provide verification that users and the network are legitimate.

Table 2-2: Key Problems with WEP (the default 802.11 Wireless LAN Security) 


\subsection{IEEE 802.1x Security Protocol}

The security structure in 802.11, including WEP and WEP-based authentication, is not designed to scale to handle large, public networks [34]. The shared key design in WEP requires the network administrator to trust many users with the same authentication credentials for the same set of APs.

802.1x, a ratified IEEE standard, solves some but not all of these problems. $802.1 \mathrm{x}$ is a port based, extensible authentication protocol. It was designed to prevent an attacker from malicious use of the network.

\subsubsection{Terminology}

Figure 2-7 (adapted from [10]) shows the supplicant, authenticator system, and authentication server in an $802.1 \mathrm{x}$ wireless network. $802.1 \mathrm{x}$ requires one authenticator per port. The controlled port shown below is not authorised and is therefore not allowing traffic.

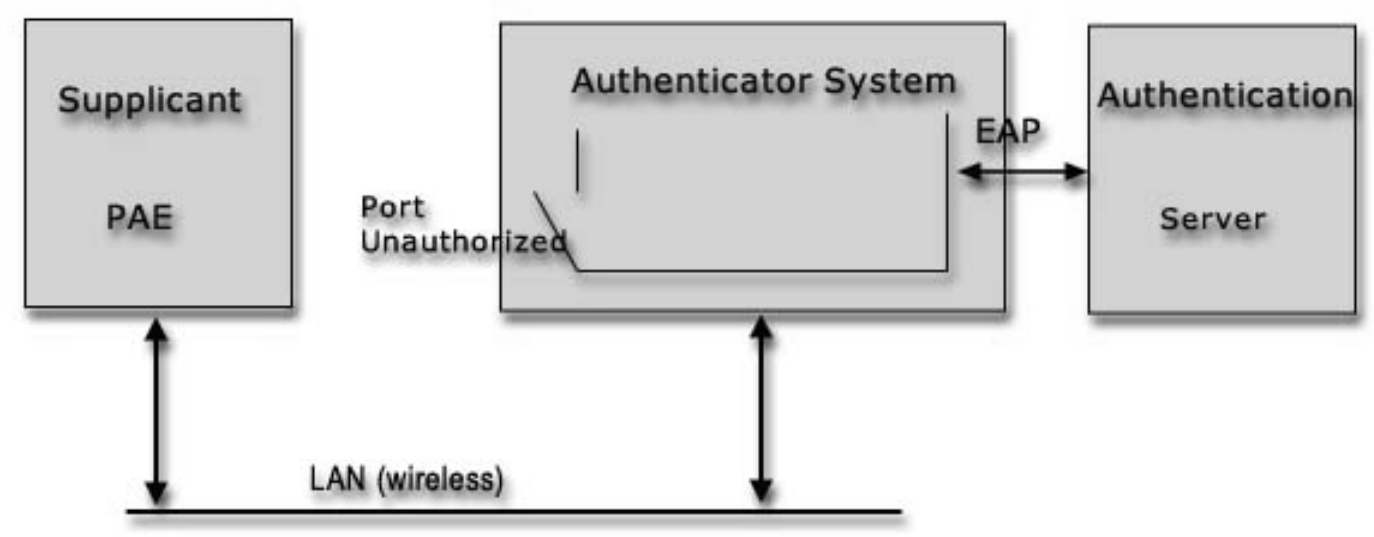

Figure 2-7: The 802.1x Basic Scenario

Port: A port is a single point of attachment to the LAN infrastructure. In the 802.11 LAN case, an AP manages logical ports. Each of these logical ports communicates one-to-one with a station's port.

Authenticator System: The authenticator enforces authentication before allowing access to services that are accessible via that port. The authenticator is responsible for communication with the supplicant as well as submitting the information received from the supplicant to a suitable authentication server. This allows the verification of user credentials to determine the consequent port authorisation state. It is important to note that the authenticator's functionality is independent of the actual authentication method. It effectively acts as a passthrough for the authentication exchange.

Supplicant: The supplicant accesses the services accessible via the authenticator. The supplicant is responsible for responding to requests from an authenticator for information, which establishes its credentials.

EAP: The Extensible Authentication Protocol (EAP) [43] is a method of conducting an authentication conversation between a user and an authentication server. Intermediate devices such as APs and proxy servers do not take part in the conversation. Their role is to relay EAP 
messages between the parties performing the authentication. 802.1x employs the EAP as an authentication framework.

Extensible Authentication Protocol over LAN (EAPOL): 802.1x defines a standard for encapsulating the EAP messages so that they can be handled directly by a LAN MAC service. This encapsulated form of EAP frame is known as EAPOL. In addition to carrying EAP packets, EAPOL also provides control functions such as start, logoff, and key distribution.

RADIUS: RADIUS is the Remote Access Dial In User Service. It is the standard way of providing Authentication, Authorisation and Accounting services to a network. Although RADIUS protocol support is optional within IEEE $802.1 \mathrm{x}$, it is expected that many $802.1 \mathrm{x}$ authenticators will function as RADIUS clients.

\subsubsection{1x Architecture}

802.1x port-based access control has the effect of creating two distinct points of access to the authenticator's attachment to the LAN. One point of access allows the exchange of frames between the system and other systems on the LAN. Often, this uncontrolled port allows only authentication messages (EAP messages) to be ex-changed. The other (controlled) point of access allows the exchange of frames only if the port is authorised.

When a host connects to the LAN port on an $802.1 \mathrm{x}$ switch the authenticity of the host is determined by the switch port according to the protocol specified by $802.1 \mathrm{x}$ before the services offered by the switch are made available on that port. Until the authentication is complete, only EAPOL frames are allowed exchanged. Once the host authentication is successful, the port switches traffic as a regular port. As previously mentioned, 802.1x was developed to address point-to-point networks. In other words, there must be a one-to-one relationship between a supplicant and an authenticator. In a wired LAN, a supplicant is directly connected to an authenticator. As shown in Figure 2-7, a workstation is directly connected to a LAN switch port. Each port on the LAN switch has an associated authenticator. The workstation gains access to the network when its supplicant authenticates to the LAN port authenticator $[10,33]$.

\subsubsection{1x in 802.11 Wireless LANs}

Applying the 802.1x structure to the 802.11 network architecture (Figure 2-8, adapted from [5]) provides a controlled wireless network with user identification, centralised authentication, and dynamic key management. Dynamic key management in an 802.1x framework rectifies the drawbacks in the WEP security mechanism by deploying per-user session keys.

The 802.1x neither excludes nor requires WEP or any other encryption algorithm. It provides a mechanism for distributing encryption key information from an AP to a client using the EAPOL-Key message. Once the station is associated with an AP, it can exchange EAP messages with the authentication server to authorise the port. Before the logical port has been authorised, it only exchanges EAP messages.

One session key can be derived for each user per session. However, if global keys (WEP keys) are used, the session key sent from the authentication server to the AP is the only used to encrypt the global key; therefore providing per-packet authentication and integrity. An EAPOW-key packet is used for the global keys. 

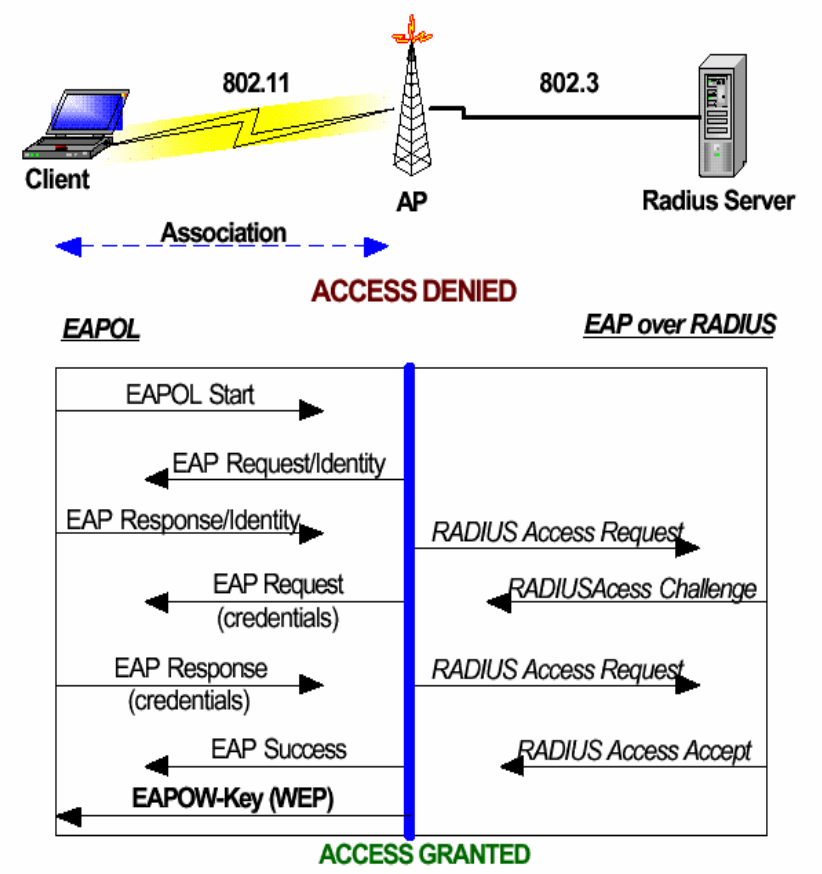

Figure 2-8: 802.1x over an 802.11 Network

\subsubsection{EAP-TLS}

EAP-TLS [36] is the most commonly implemented EAP type for wireless LANs. It provides secure mutual authentication using digital certificates. When a client requests access, the response from the authentication server is a server certificate. The client has a certificate, signed by a trusted certificate authority, which has been preconfigured by the network administrator. The client will reply to the authentication server's challenge with its own certificate and validates the server certificate at the same time. Based off the certificate values, the EAP-TLS algorithm can derive dynamic WEP keys, and the authentication server will send the client the WEP key for use during that session.

Certificate-based algorithms such as EAP-TLS are highly secure, as it is nearly impossible to forge a certificate digitally signed by a certificate authority. On the other hand, the management of certificates can be complex and expensive [1]. Thus, depending on the scale of an organisation's network, administrative burdens might outweigh the security advantages. Figure 2-9 (adapted from $[5,58]$ ) illustrates the process.

\subsection{Wireless Performance}

Security is a property of an entire system and every decision must be examined with security in mind [26]. There have been many evaluation studies of IEEE 802.11 wireless network performance; however, little attention has been paid to the effects of implementing security on performance. Some relevant evaluation studies have been described in this section. Chapter 3 also introduces some related work in this area in order to justify the design and parameters used in this research. 

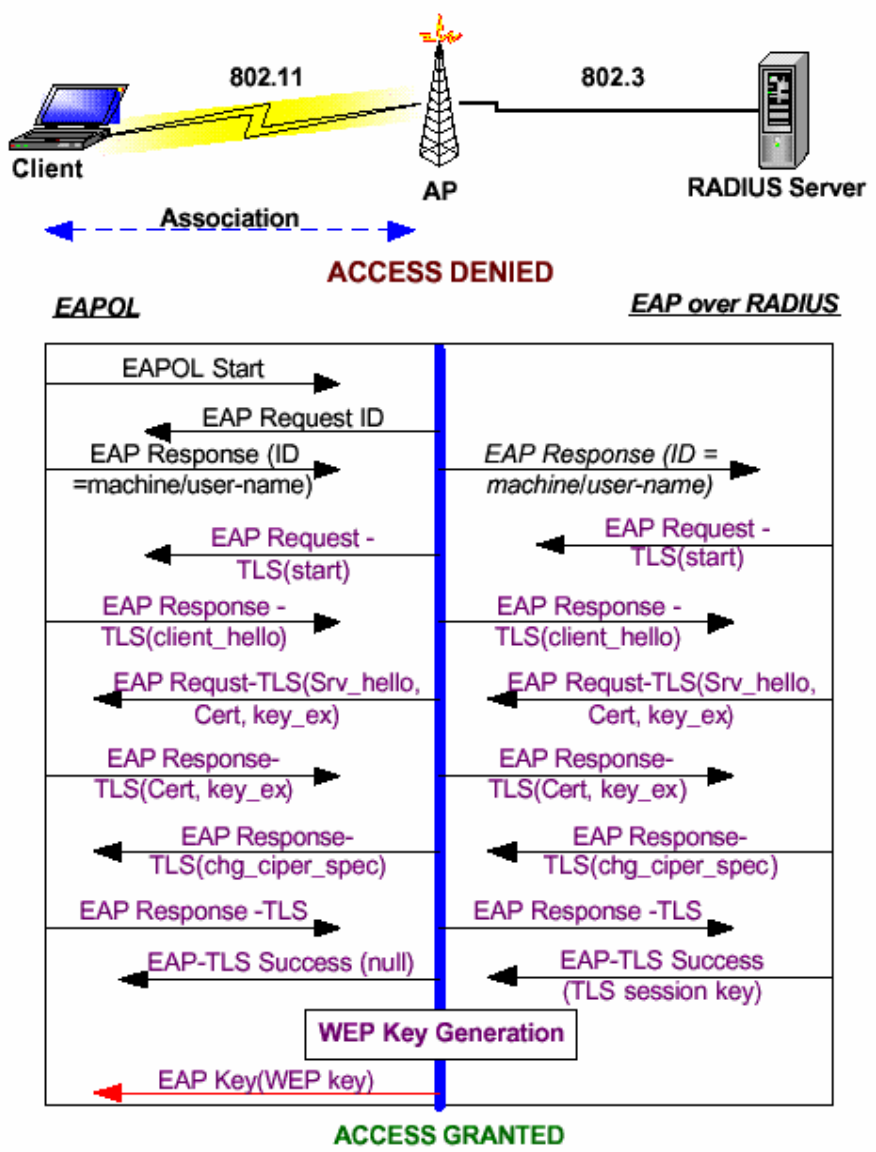

Figure 2-9: EAP-TLS Authentication Process

Amaro et al. [41] evaluated the performance of wireless networks and concluded that, the larger the packet size, the higher the effective rate. The collision avoidance mechanism of $802.11 \mathrm{~b}$ protocol confirmed this increase, as traffic overheads introduced by the control frames RTS/CTS (see Section 2.2.4) and the ACK frames diminished for larger packets.

A number of tests, described by [39], conducted on the 802.11 wireless LANs to measure performance characteristics (throughput and response time) of the MAC layer under various network loads. The results showed that the buffering and fragmentation of data frames can seriously influence the performance of an 802.11 wireless LAN. Although the length of a data frame and the bit rate of the wireless transceiver also affect the wireless LANs transmission capabilities, its performance is generally unaffected by the type of frame addressing and the use of reservation frames such as RTS and CTS.

Chen [11] carried out an experiment to compare the coverage area and performance between 802.11b (11 Mbps) and 802.11a (54 Mbps). The study showed that 802.11a provides 2-to-5times better data-link rate and throughput performance in the same range $(77 \mathrm{~m})$ as $802.11 \mathrm{~b}$. Chen also compared the trade-off between performance and costs in terms of range and total system capacity. 802.11a offered better system capacity with fewer cells (APs).

Empirical results from [55] demonstrated that different modes of 802.11 wireless LAN and Ethernet frame size were crucial factors in the determination of a wireless LAN's transmission capabilities. The throughput of a wireless LAN increased as the frame length 
increased and as the amount of broadcast traffic decreased. Furthermore, the authors suggested that the mean response times for both wired and wireless LANs were similar, with an inter-frame delay of $10 \mathrm{~ms}$ or more.

An empirical characterisation of the instantaneous throughput of a station in an $802.11 \mathrm{~b}$ Wireless LAN, as a function of the number of competing stations sharing the AP, was presented in [40]. The results showed that as the number of stations increases, the overall throughput decreases and its variance increases.

Kamerman et al. [42] evaluated the throughput of 802.11 wireless LANs with respect to various kinds of overhead. The impact of several sources of overhead was modeled. Sources included gap time, preamble, physical layer, MAC layer and TCP/IP header fields, ACK and request frames. After measurement of the net throughput and detailed monitoring of actual exchange of frames, this modeling was refined. A close fit was found between the results for IEEE $802.11 \mathrm{~b}$ obtained from this model and as measured using currently available $2.4 \mathrm{GHz}$ products.

A recent study [5] evaluated the security performance of an 802.11 wireless network, by measuring the throughput and response time of HTTP and FTP traffic types in an unsaturated, simple point-to-point architecture. The results showed that the stronger the security mechanism implemented, the poorer the network performance. The study recommended using multiple clients (in order to experiment with congestion in a secure environment), and looking at a wide range of traffic types. These recommendations were used as the basis for this research. The research also evaluated the performance of wireless LANs at the packet level, as described in next chapter. 


\section{Chapter 3}

\section{Experiments}

\subsection{Aim}

This aim of this research is to investigate the performance and security issues of $802.11 \mathrm{~b}$ wireless LANs with multiple clients, hence demonstrating contention in a secure environment.

The following issues are addressed in particular:

- How do different security mechanisms affect the performance (delay and throughput) of a congested wireless LAN with multiple clients?

- What are the effects of different packet lengths on the performance of wireless LANs using different security mechanisms?

․ What is the impact of security on different traffic types?

- How does the performance of a secure network vary by adding more clients?

\subsection{Method}

\subsubsection{Design Considerations}

There were a few design decisions made before carrying out the experiments. The main three were related to security layers, the traffic generator and the performance measurements used in the experiments. They are described below:

\section{Defining Security layers}

As part of the research objectives, we wanted to experiment with the effect of WEP authentication and encryption as well as IEEE $802.1 \mathrm{x}$ authentication. The security layers, therefore, had to be defined in a way that they would include all the possibilities.

\section{Traffic Generator}

As mentioned in Section 3.1, this research focuses on the performance evaluation of congested wireless LANs. The generator had to be flexible and capable of overloading such networks. The specific requirements we had in mind for choosing a traffic generator were:

a Suitable for wireless networks

a Capable of overloading an 802.11 LAN

a Allowing the user to change the size and inter-packet delay

- Allowing the user to select the generation algorithm 


\section{Measuring performance}

Many factors affect network performance and some of them interact to provide overall performance results. Performance results vary depending on the choice of hardware device, software application and network topology [5]. Some of the performance measurements are $[49,50]$ : Response time, Throughput, Coverage area, Mobility, Bandwidth, Latency, Radio signal strength, etc. Response time and Throughput were measured in this research to provide a comprehensive view of the network performance ${ }^{18}$. They are defined as follows:

a Response time: the total time required traffic to travel between two points. It includes the time of dial-up connection establishment, security negotiation time between the server and the clients and the actual data transfer.

- Throughput: the total number of bytes transmitted over the network in a given time (response time).

\subsubsection{Configuration of Wireless LAN system}

It was decided to use Windows-based operating systems, since Windows XP has a built-in implementation of the IEEE 802.1x authentication protocol [51]. As shown in Figure 3-1, the experiments were conducted using:

- One Server

o Windows 2000 Advanced server

o $1.4 \mathrm{GHz}, 512 \mathrm{MB}$ RAM, Orinoco AP-2000 software

a Three Clients

- Windows XP Professional

- $1.4 \mathrm{GHz}, 512 \mathrm{MB}$ RAM, Orinoco USB client and ORiNOCO Wireless LAN Gold Cards

\section{a Access Point}

o Lucent Orinoco AP-2000

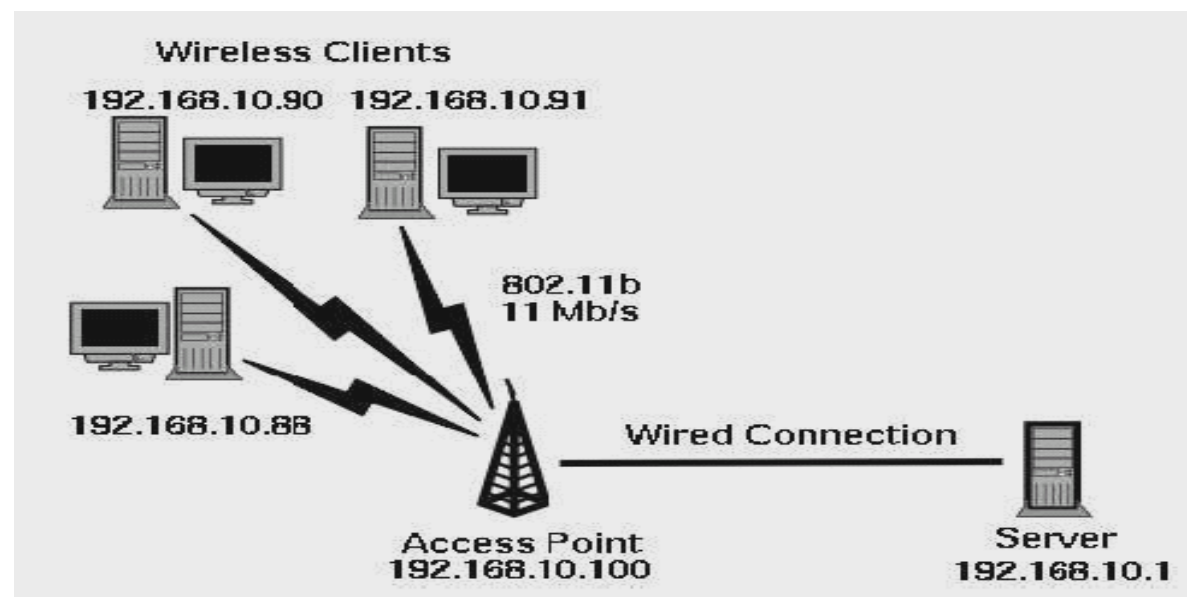

Figure 3-1: Experimental set up

\footnotetext{
${ }^{18}$ These two parameters have been measured in other wireless performance studies, such as [39].
} 
Transmission speed was $11 \mathrm{Mbps}$ wireless connections between the AP and the clients and $100 \mathrm{Mbps}$ Ethernet connections between the AP and the server. Ethereal ${ }^{19}$ Network Analyser was used to capture live network statistics. The measurements were collected from the server. Appendix $\mathrm{B}$ provides instructions on implementing security mechanisms ${ }^{20}$ and further enhancing the system architecture used in [5] to support multiple clients.

\subsubsection{Security Layers}

The following eight security layers were chosen to present a hierarchical order of the security mechanisms available from both IEEE 802.11 and IEEE 802.1x standards (For more details about each protocol, see Sections 2.4, 2.5):

1. No security: this is the default security setting provided by vendors. There is no security mechanism activated with default configuration.

2. MAC address authentication: this layer provides MAC address authentication carried out at the AP.

3. WEP authentication: the shared key authentication method specified in the 802.11 standard is used.

4. WEP authentication with 40-bit WEP encryption: this layer combines the encryption algorithm to provide data privacy.

5. WEP authentication with 128-bit WEP encryption: the 128-bit shared key used is proprietary-based (in the case of Lucent).

6. EAP-TLS authentication: this is the PKI-based authentication method supported by $802.1 \mathrm{x}$, using digital certificates to authenticate the $u^{21} \mathrm{er}^{21}$.

7. EAP-TLS with 40-bit WEP encryption: the combined effect of these tools provides the strongest layer of encryption and authentication using per-session keys.

8. EAP-TLS with 128-bit WEP encryption: this layer is the same as above using 128-bit keys.

The first five security layers are consistent with the 802.11 standard. Security layers 6 to 8 are provided by the $802.1 \mathrm{x}$ standard.

\subsubsection{1x Model Implementation}

The $802.1 \mathrm{x}$ model consisted of the 802.11 access mechanism using open and shared key authentication, WEP encryption, and the $802.1 \mathrm{x}$ port-based authentication. By combining $802.1 \mathrm{x}$ with 802.11 protocols (as security layers 6 to 8 ), the model provided a controlled wireless network with user identification, centralised authentication, and dynamic key management.

For security layers 6 to 8 , a RADIUS server was used to provide dynamic key management and centralised authentication (see Figure 3-2, where the server and one of the clients shown).

\footnotetext{
${ }^{19} \mathrm{http}: / /$ www.ethereal.com/

${ }^{20}$ In this report security layer and security mechanism represent the same concept.

${ }^{21}$ For further details, refer to section 2.5.4.
} 
The authentication method chosen for the experiments was EAP-TLS. The 802.1x model does not support end-to-end security, because privacy and confidentiality were only ensured on the wireless link by the WEP, but not enforced on the wired counterparts.

Wireless users were treated as if they existed in one sub-network of an organisation's intranet. Specific IP addresses were assigned to the wireless users, AP and different components of the server.

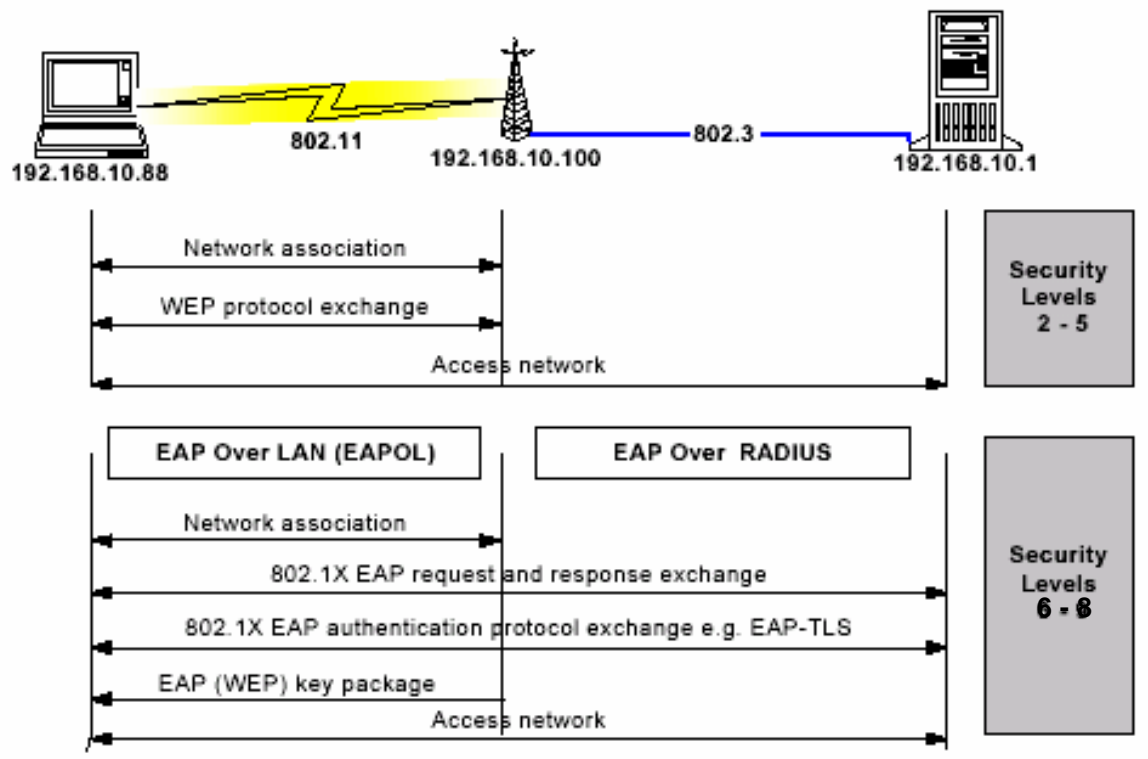

Figure 3-2 802.1x Model Logical Flow

The RADIUS server and certificate authorities were added to the basic network structure to provide the $802.1 \mathrm{x}$ authentication support (Figure 3-3). The RADIUS server supported wireless user sign-on, and a certificate authority was used to issue certificates to users for EAP-TLS authentication ${ }^{22}$.

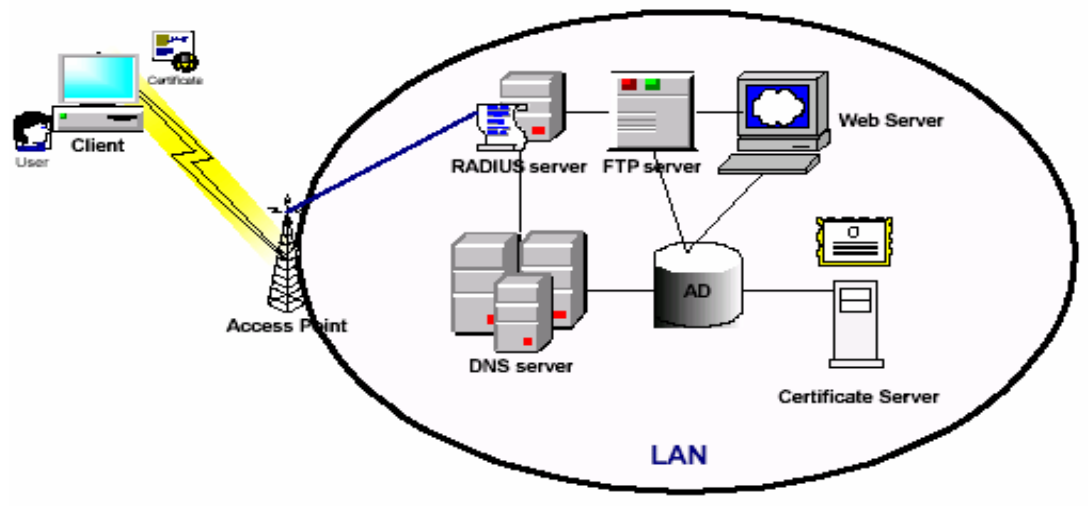

Figure 3-3 802.1x Model Implementation

22 This section is a modified version of a section from [5]; the approach taken in implementing $802.1 \mathrm{x}$ model by this research and [5] are relatively similar. 


\subsubsection{Traffic generator}

We were interested in the ability of a wireless LAN to transfer IP packets in a predefined number, size, content and bandwidth in order to measure the variation in performance when security mechanisms are implemented.

After spending a considerable amount of time searching for an appropriate traffic generator, IP Traffic ${ }^{23}$ tool was found. It was felt that this generator had met all the requirements we had in mind (see Section 3.2.1). IP Traffic is a software-testing tool that is designed for both fixed and wireless IP networks and runs on Windows platforms. It can generate, receive, capture and replay IP traffic, and measure end-to-end performance and Quality of Service over any IP fixed or mobile network. The generator can manage several simultaneous IP connections (see Figure 3-4); however, we only used one active connection from each client to model a more realistic situation. We used the real time statistics generated by IP Traffic as well as the data collected by Ethereal on the server side to evaluate the performance of the wireless network.

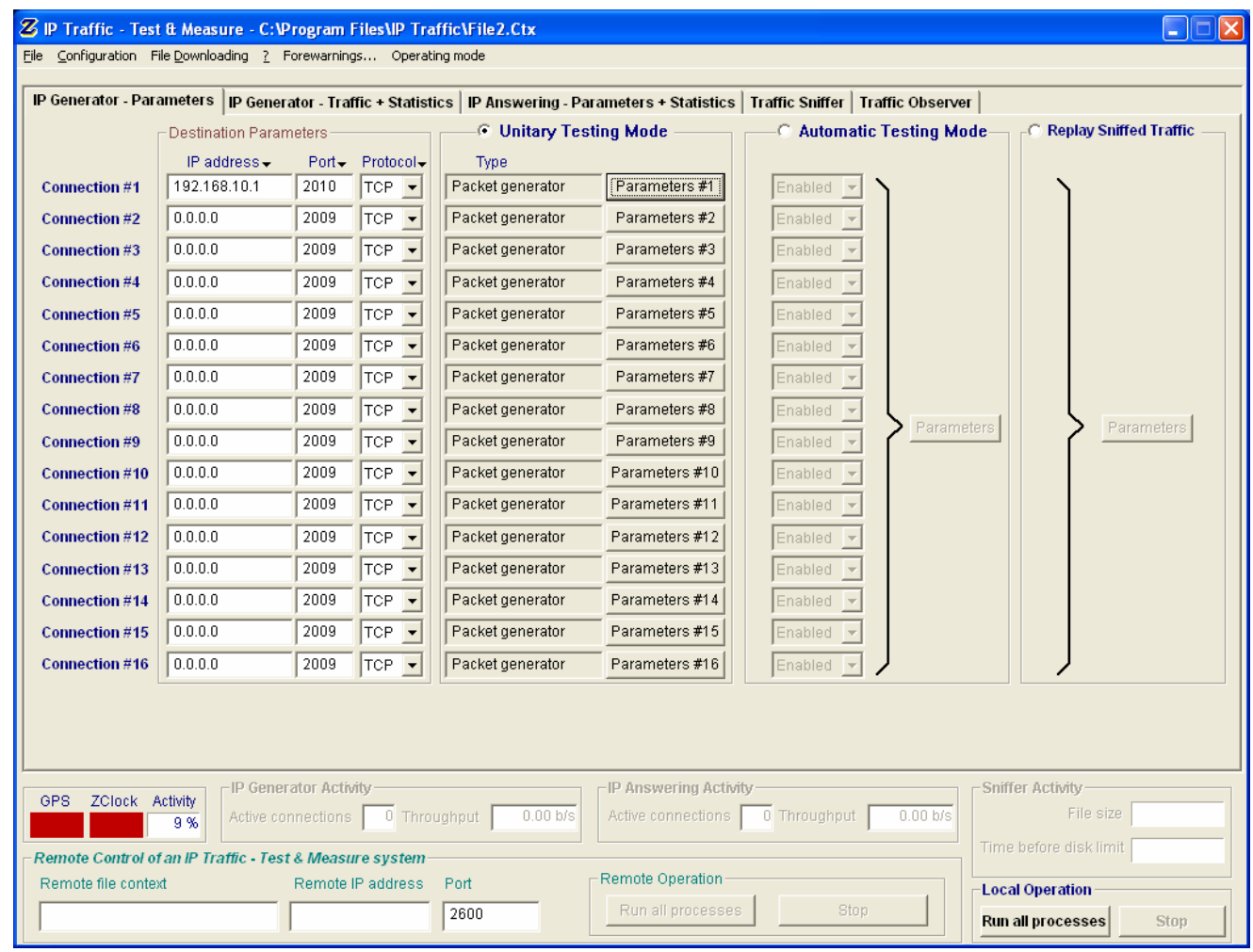

Figure 3-4: IP traffic tool on client side

The following describes and justifies the parameters specified in the generator for the duration of the experiments (see Figure 3-5):

\section{Total number of packets}

Preliminary experiments showed that the choice of packets number did not affect the trends observed in the results. Ranges between 10000 and 60000 were tried and 43000 was selected as an arbitrary value between the two.

\footnotetext{
${ }^{23}$ http://www.zti-telecom.com/pages/iptraffic-test-measure.htm
} 


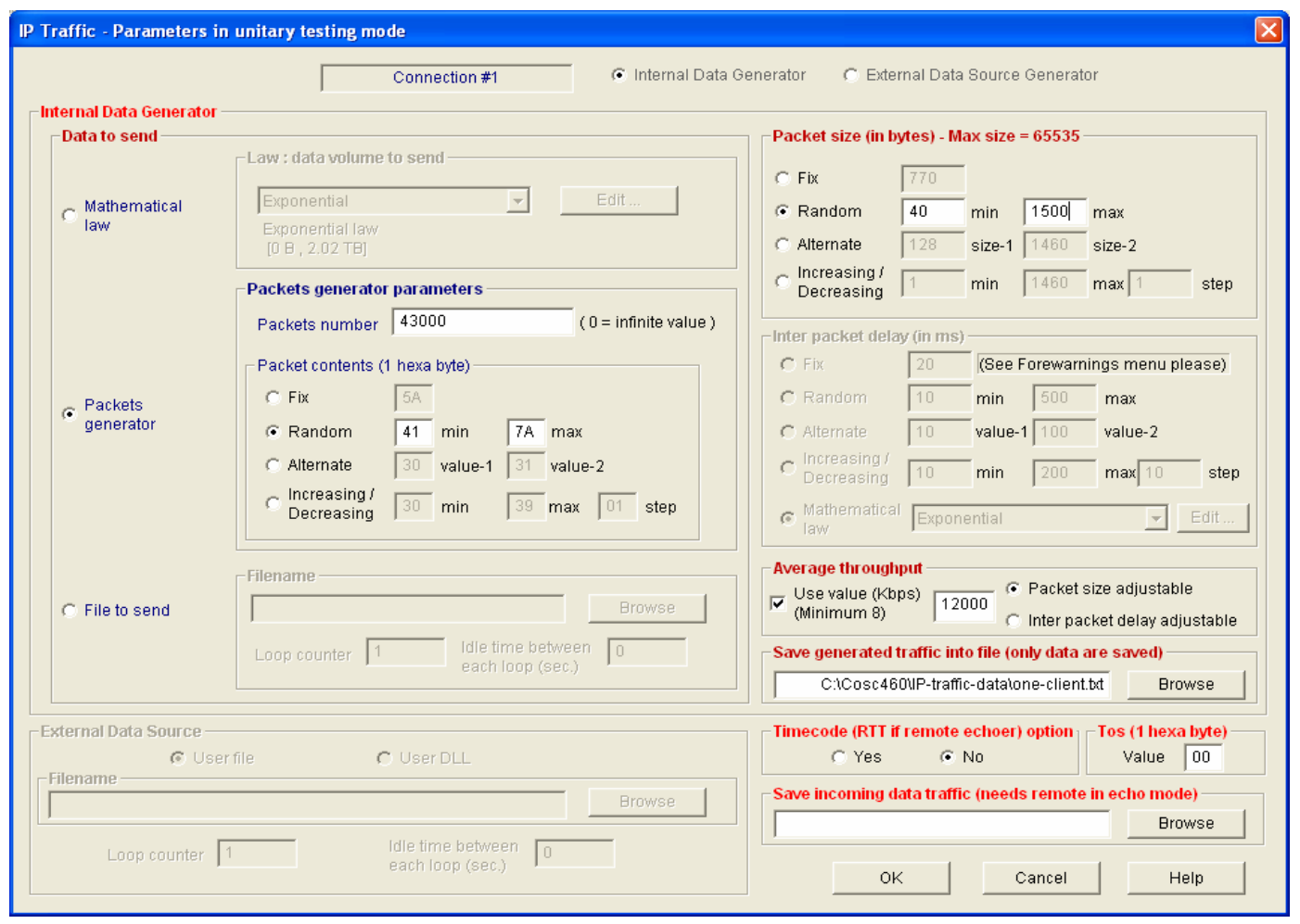

Figure 3-5: Parameters specified for each connection

\section{Outgoing bandwidth}

The incoming bandwidth of an $802.11 \mathrm{~b}$ Access Point is $11 \mathrm{Mbps}$ (according to standards). Since we were interested in the behaviour of networks under congestion, we decided to set the outgoing bandwidth of each client to be $12 \mathrm{Mbps}$-well in excess of the published 802.11 maximum to ensure a congested scenario.

\section{Traffic type}

It was decided to experiment with TCP and UDP protocols, as these protocols form the basis of all the applications running on the IP protocol stack. Other studies have evaluated the performance of TCP and UDP protocols over wireless networks (e.g. [40, 44, 52]). However, none of them took the impact of different security mechanism into account.

\section{Content}

Content of each packet was decided to be random. This parameter was not of any importance in this research.

\section{Packet length}

It was decided to set the packet length to be a random integer uniformly distributed over the interval $[40,1500]$ in the first set of experiments. The main advantage of this traffic model is that it allows exploring the full range of packet sizes [46]. It was decided to choose 40 and 1500 bytes as boundaries of IP packet sizes, as these numbers have been used in previous woks $[44,45,48]$. In addition, these values were chosen after capturing live traffic on a COSC LAN for 3 days $^{24}$ using Ethereal and considering the packet sizes. Random generation

\footnotetext{
${ }^{24}$ This was done on July 8-10, 2003.
} 
of packet lengths compared with using fixed sizes (in the first set of experiments), provided us with more realistic situation.

In the second part of experiments (studying throughput as a function of packet sizes), four fixed packet sizes (i.e. 100, 500, 1000, 1500) were selected and the experiments were conducted using each value at a time. These values have been used in other performance studies such as $[44,52]$.

\subsection{Procedure}

As described in Section 3.1, this research aims to evaluate the effect of different security mechanisms and packet sizes on the performance of a congested wireless LAN with multiple clients.

In the first set of experiments, the throughput and response times of two traffic types (TCP and UDP) were measured under different security mechanisms. The experiments were then repeated for two and three clients to study the impact of adding more clients. Two different bandwidths were defined: $12000 \mathrm{~kb} / \mathrm{s}$ (to represent a congested network) and $500 \mathrm{~kb} / \mathrm{s}$ (to represent an unsaturated network). It was decided to set the packet length to be a random integer uniformly distributed over the interval [40, 1500], as stated in the previous section. The security layers, traffic generator and the system configuration used during the experiments are detailed in Section 3.2. Figure 3-6 illustrates the configuration of traffic generator on the server side, where the clients' IP addresses were manually allocated. Appendix B provides instructions on setting up the system.

In the second set of experiments, the throughput was studied as a function of different packet sizes, under different security mechanisms. The packet sizes were divided into four fixed numbers, i.e. $100,500,1000,1500$, as described before. The experiments were conducted using one client and three different security mechanisms ${ }^{25}$, and throughput was measured for both TCP and UDP traffic types. Results are discussed in Section 3.4.

Each experiment was repeated eight times. Due to system factor influences (memory caching and disk pages), it was decided to discard the first three results, to exclude system factors.

\begin{tabular}{|c|c|c|c|c|c|c|c|c|c|}
\hline File Configuration & File Downloc & ading ? & For & ewarning & s... Ope & erating & mode & & \\
\hline IP Generator - P & arameters & IP Gen & erat & or - Trat & fic + Stat & tistic: & IP Ansv & $\lg -1$ & \\
\hline \multirow[b]{2}{*}{ Connection \#1 } & {$\left[\begin{array}{l}\text { Connecte } \\
\text { Source IP }\end{array}\right.$} & $\begin{array}{l}\text { d Remo } \\
\text { address }\end{array}$ & Connected Remote & \multirow{2}{*}{$\begin{array}{l}\text { Port- } \\
2010\end{array}$} & Protoco & & Receiving Working Mode & king & \\
\hline & 192.168 .1 & 10.88 & $\nabla$ & & UDP & + & Absorber & $\nabla$ & \\
\hline Connection \#2 & 192.168 .1 & 10.91 & $\nabla$ & 2011 & UDP & $\nabla$ & Absorber & 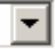 & \\
\hline Connection \#3 & 192.168 .1 & 10.90 & 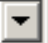 & 2012 & UDP & $\nabla$ & Absorber & $\nabla$ & \\
\hline Connection \#4 & 255.255 .2 & 255.255 & $\nabla$ & 2009 & $\mathrm{TCP}$ & 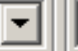 & Absorber & 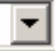 & \\
\hline Connection \#5 & 255.255 .2 & 255.255 & 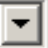 & 2009 & TCP & 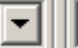 & Absorber & 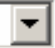 & \\
\hline Connection \#6 & 255.255 .2 & 255.255 & - & 2009 & TCP & 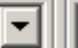 & Absorber & $\checkmark$ & \\
\hline
\end{tabular}

Figure 3-6: Configuration set up on server side

\footnotetext{
${ }^{25}$ Chosen from the eight mechanisms, listed in Section 3.2.3.
} 


\subsection{Results}

The experiments followed the eight security layers described in section 3.2.3. An infrastructure mode of operation and a single cell were used with three clients. Performance measures were gathered by running five repetitive tests at each security configuration. Experiments evaluating the performance of TCP were separated from UDP's and each set was conducted for different number of clients. Results were collected through log files generated by the traffic generator and the Ethereal monitoring tool. Data were analysed, at the corresponding $95 \%$ confidence interval.

\subsubsection{Effect of security mechanisms on performance}

In the first part of the experiments, the bandwidth was set to $500 \mathrm{~Kb} / \mathrm{s}$ to represent a lightly loaded network (in other words, normal situation when the network is not congested). Figure 3-7 illustrates the throughput of TCP and UDP traffic types under different security layers, discussed in Section 3.2.3. These results confirmed the general trends reported in [5], meaning that the stronger the security mechanism implemented, the poorer the network performance.

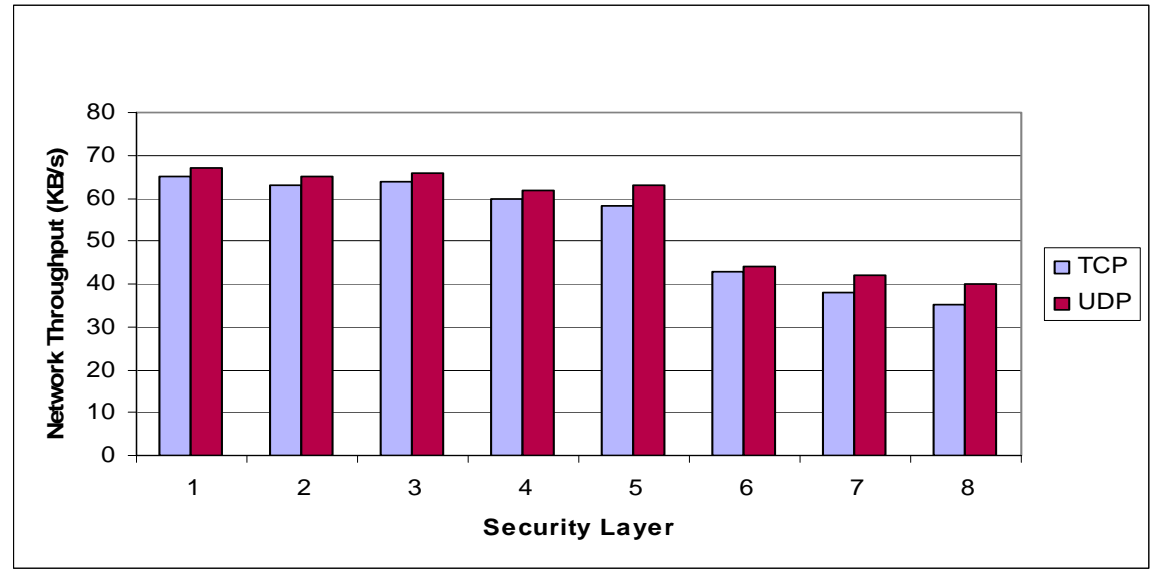

Figure 3-7: Throughput of TCP, UDP traffic in an unsaturated network

Figure 3-8 and 3-9, on the other hand, illustrate the throughput and (per-packet) response times of TCP and UDP traffic types when the network is congested (bandwidth is set to 12 $\mathrm{Mb} / \mathrm{s})$.

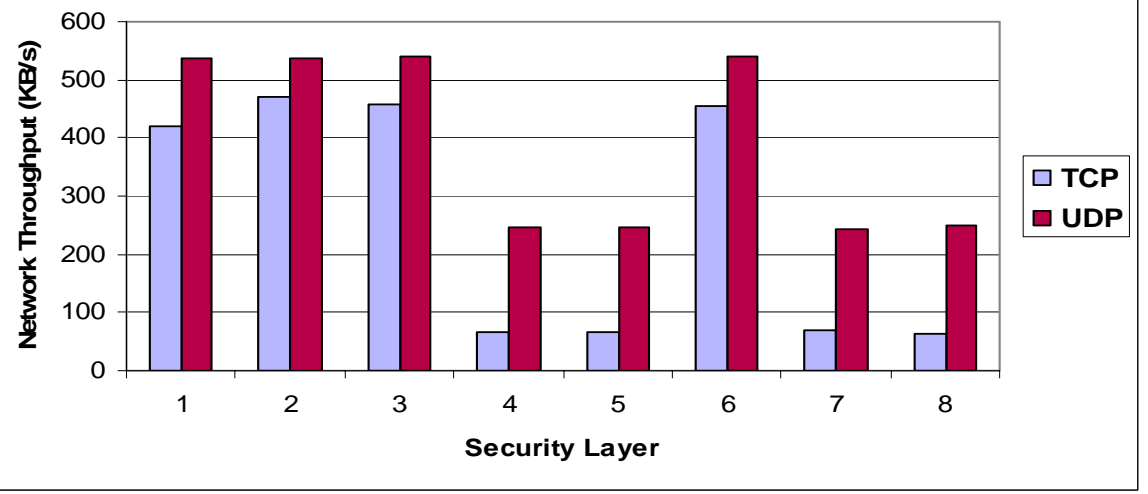

Figure 3-8: Throughput of TCP, UDP traffic in a congested network 
As the graphs show, the performance of a congested network at security layers 4, 5, 7 and 8 (WEP encryption is in place) is significantly less than the performance of the network at security layers 1, 2, 3 and 6 . The security layers 4, 5, 7 and 8 decrease the TCP throughput by $86.1 \%$ and the UDP throughput by $54.3 \%$ (on average). In addition, they increase the TCP response time by $86.2 \%$ and UDP response time by $51.9 \%$. The results show that in congested networks, the overhead produced by encrypting each individual packet (implemented at security layers 4, 5, 7 and 8), is significantly higher than applying more advanced authentication methods, such as EAP-TLS protocol implemented at security layer $6^{26}$.

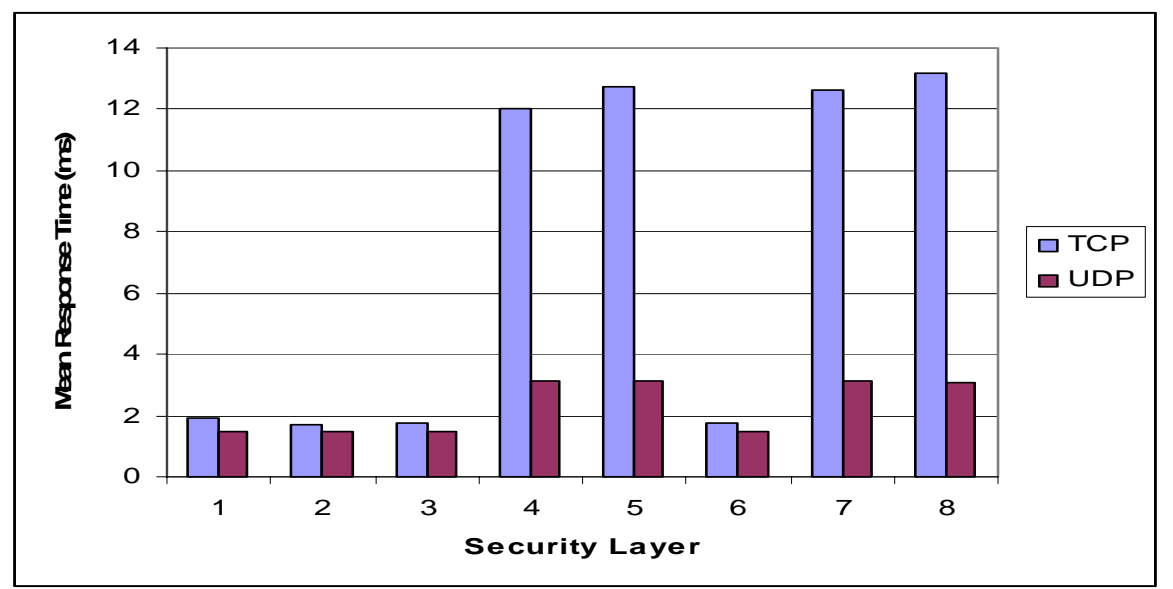

Figure 3-9: Per-packet response times of TCP, UDP traffic in a congested network

The performance of TCP and UDP can also be compared. Since the TCP protocol uses a congestion control mechanism, it is significantly slower than the UDP protocol in congested networks, especially when WEP encryption is applied. The TCP throughput is $21.6 \%$ of UDP throughput at security layers 4, 5, 7 and 8 and $85.5 \%$ of UDP throughput at security layers 1 , 2,3 and 6.

Table 3-1 and 3-2 show the mean and standard deviation of TCP and UDP throughput and response times when the wireless LAN is congested.

\begin{tabular}{|c|r|r|r|r|}
\hline $\begin{array}{c}\text { Security } \\
\text { Layer }\end{array}$ & \multicolumn{2}{|c|}{ TCP } & \multicolumn{2}{c|}{ UDP } \\
\hline 1 & 447.5200 & 7.3095 & 535.6960 & \multicolumn{1}{c|}{ SD } \\
\hline 2 & 470.7520 & 4.3070 & 535.0120 & 1.0143 \\
\hline 3 & 464.4460 & 11.5685 & 536.5260 & 4.0540 \\
\hline 4 & 67.2940 & 1.7635 & 245.4660 & 0.3958 \\
\hline 5 & 63.7380 & 1.8943 & 245.6100 & 2.0043 \\
\hline 6 & 454.6480 & 0.4346 & 539.4560 & 5.4398 \\
\hline 7 & 64.3000 & 2.1949 & 244.3340 & 0.7835 \\
\hline 8 & 61.5300 & 1.9546 & 245.8260 & 3.2859 \\
\hline
\end{tabular}

Table 3-1: The Mean and SD of TCP and UDP throughput under different security mechanisms

The security layers significantly differ from each other in their effects on the throughput of the network for TCP $(F(7,28)=8558.155, p<.001)$ and $\operatorname{UDP}(F(7,28)=14155.207, p<.001)$

\footnotetext{
${ }^{26}$ This is not the case in unsaturated networks (shown in Figure 3-7), as the network performance at layer 6 (EAP-TLS authentication) is lower than layer 5 (WEP encryption with 128-bit keys).
} 
traffic types. The security layers also significantly differ from each other in their effects on the response time of the network, for both $\operatorname{TCP}(F(7,28)=2439.143, p<0.001)$ and $\operatorname{UDP}(F(7$, $28)=9103.760, p<0.001)$ traffic. The data that are used for ANOVA analysis, are based on Appendix A Captured Data ${ }^{27}$.

\begin{tabular}{|c|r|r|r|r|}
\hline $\begin{array}{c}\text { Security } \\
\text { Layer }\end{array}$ & \multicolumn{2}{|c|}{ TCP } & \multicolumn{1}{c|}{ UDP } \\
\hline 1 & 77.2240 & 0.7888 & 64.4800 & \multicolumn{1}{c|}{ SD } \\
\hline 2 & 73.5660 & 0.7748 & 64.5200 & 0.2233 \\
\hline 3 & 75.2880 & 1.9601 & 64.6400 & 0.6479 \\
\hline 4 & 520.7700 & 13.9539 & 134.0740 & 0.1266 \\
\hline 5 & 546.7900 & 20.1678 & 134.3780 & 0.9191 \\
\hline 6 & 76.4840 & 0.0568 & 63.8280 & 0.5364 \\
\hline 7 & 549.8400 & 20.6787 & 134.7220 & 0.4132 \\
\hline 8 & 573.6260 & 9.5460 & 131.7140 & 2.0734 \\
\hline
\end{tabular}

Table 3-2: The Mean and SD of TCP and UDP response times under different security mechanisms

\subsubsection{Effect of adding more clients}

To evaluate the performance of the network in a secure multi-client environment, the experiments were repeated using two and three clients. Table 3-3 shows the average perstation throughput for UDP traffic. Over all security layers, the average throughput of each station decreased by $49.5 \%$ when the experiments were conducted using two clients and $66.5 \%$ when using three clients. ${ }^{28}$

\begin{tabular}{|c|r|r|r|}
\hline \multirow{2}{*}{$\begin{array}{c}\text { Security } \\
\text { Layer }\end{array}$} & \multicolumn{3}{|c|}{ Per - station throughput (KB/s) } \\
\hline 1 & 1 Client & 2 Clients & 3 Clients \\
\hline 2 & 535.68 & 279.55 & 176.79 \\
\hline 3 & 535.44 & 273.07 & 178.97 \\
\hline 4 & 539.37 & 269.6 & 185.77 \\
\hline 5 & 245.61 & 120.37 & 78.89 \\
\hline 6 & 245.28 & 122.88 & 84.14 \\
\hline 7 & 540.13 & 273.06 & 178.28 \\
\hline 8 & 244.45 & 121.73 & 79.61 \\
\hline & 248.17 & 122.09 & 84.78 \\
\hline
\end{tabular}

Table 3-3: Average per-station throughput for UDP traffic

When WEP encryption was not enabled (under security layers 1, 2, 3 and 6), UDP packet loss rates were $3.2 \%$, and $0.6 \%$ for TCP packet. The UDP drop rate increased by $3.6 \%$ when WEP encryption was enabled (security layers $4,5,7$ and 8 ), while only increasing $0.2 \%$ for TCP traffic. On average, the observed results were increased by $0.4 \%$ when the number of clients was increased to three.

Our experiments validate the results reported in [40], which presented an empirical characterisation of the instantaneous throughput of a station in an $802.11 \mathrm{~b}$ Wireless LAN, as a function of the number of competing stations sharing the AP. The results showed that as the number of stations increases, the overall throughput decreases and its variance increases.

\footnotetext{
${ }^{27}$ Appendix A provides the data collected for one client.

${ }^{28}$ Same results were observed for TCP traffic type.
} 
However, that study did not take into account the effect of having different security mechanisms ${ }^{29}$ in place.

\subsubsection{Effect of various packet sizes on performance}

As described in Section 3.3, four fixed packet sizes, i.e. 100, 500, 1000, 1500 bytes were chosen in order to evaluate their impacts on the throughput of a congested wireless LAN under different security mechanisms.

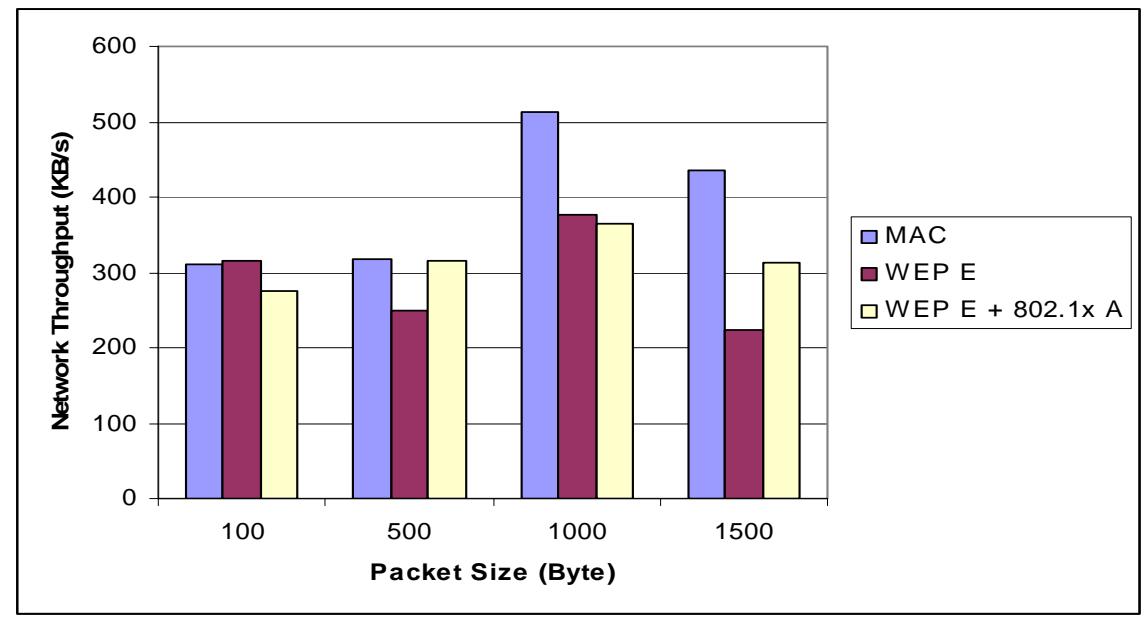

Figure 3-10: TCP throughput for different packet sizes

As Figure 3-10 shows, the throughput of TCP traffic is maximum when the packet size is set to 1000 bytes and this is the case for the three security layers chosen (MAC authentication, WEP Encryption with 128-bit keys, and WEP Encryption combined with 802.1x authentication).

Figure 3-11 shows similar results for UDP throughput. The throughput has the highest value when the packet size is set to 1000 bytes, at first and third security layer. When the $2^{\text {nd }}$ security layer is implemented (WEP encryption) packet size of 500 and 1000 bytes have almost the same throughput, with $0.1 \mathrm{~KB} / \mathrm{s}$ difference (as shown in Table 3-5).

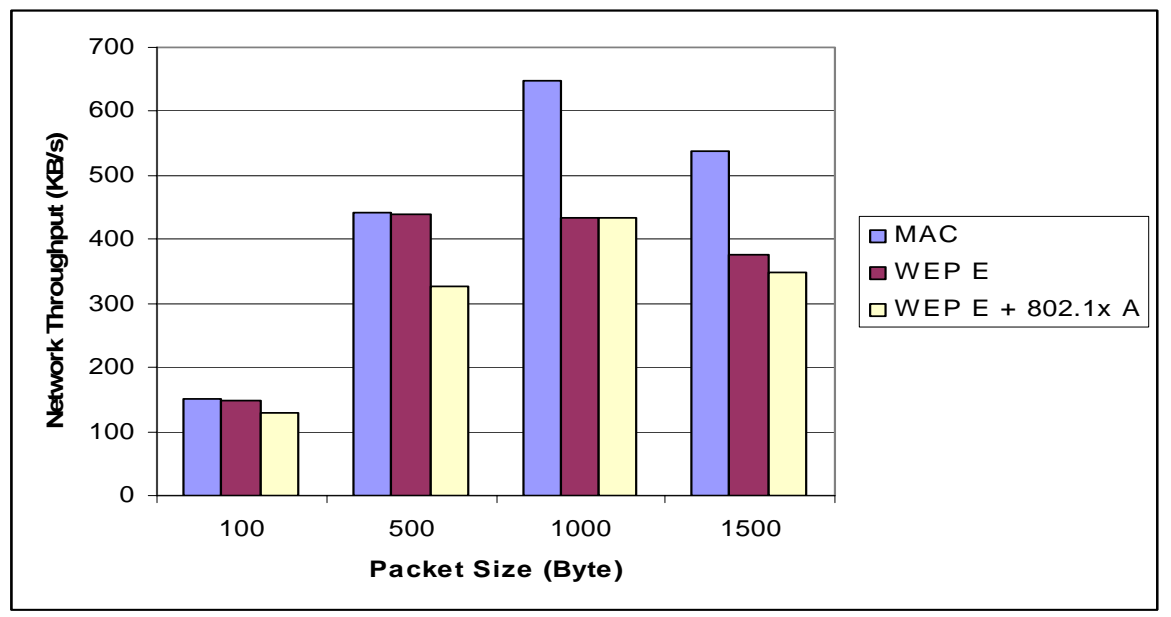

Figure 3-11: UDP throughput for different packet sizes

\footnotetext{
${ }^{29}$ As detailed in section 3.2.3
} 


\begin{tabular}{|r|r|r|r|}
\multicolumn{1}{l|}{$\begin{array}{l}\text { Packet } \\
\text { Size (Byte) }\end{array}$} & \multicolumn{1}{|l}{$\begin{array}{l}\text { MAC } \\
\text { Authentication }\end{array}$} & \multicolumn{1}{l|}{$\begin{array}{l}\text { WEP Encryption + } \\
\text { Encryption }\end{array}$} & $\mathbf{8 0 2 . 1 x}$ Authentication \\
\hline 100 & 150.81 & 147.69 & 130.18 \\
\hline 500 & 443.29 & 439.7 & 327.26 \\
\hline 1000 & 648.82 & 439.8 & 434.67 \\
\hline 1500 & 538.01 & 375.59 & 347.54 \\
\hline
\end{tabular}

Table 3-5: UDP throughput for different packet sizes

\subsection{Discussion}

Network performance was observed to significantly decrease in a congested situation when WEP encryption was applied. With WEP encryption in place, the TCP throughput decreased by $86.1 \%$ and the UDP throughput by $54.3 \%$ (on average). Three factors contribute to such a change in performance, with the third being the most influential:

- WEP Encryption: WEP uses an RC4 stream cipher in order to provide confidentially and integrity ${ }^{30}$. When WEP is in use, the fame body expands by eight bytes. Four bytes are used for a frame body IV header, and four are used for the ICV trailer [14]. These additional eight bytes do not affect the performance in an unsaturated network, as reported by [5] and confirmed in section 3.4.1 of this report. However, when the network is congested, there is not enough bandwidth available. The packets will be dropped at the AP, due to their additional length. The performance might, therefore, be decreased

- WEP resynchronisation: The loss of a single bit of a data stream encrypted under RC4 causes the loss of all the data following the lost bit. This is because data loss desynchronises the RC4 encryption and decryption engines [21]. The resynchronisation problem gets only worse as more bits become lost. Since 802.11 often drops entire packets, they will have to be resent by the client sides, which will result in performance degradation of congested networks. ${ }^{31}$ The WEP architecture accommodates itself to loss by reinitialising the cipher key schedule on every data frame.

- Hardware and software implementation: WEP encrypts frames as they traverse the wireless medium [14]. In other words, the encryption is done between the client machines and the AP. The wireless cards used in the experiments [53], encrypt/decrypt the packets at the firmware and the AP [54], encrypt/decrypts them at the hardware. When there is not enough bandwidth in the network, the buffer at the AP fills up and it keeps dropping the packets, because the CPU at the AP is too congested to process any additional data.

According to these results, in congested wireless networks, the overhead produced by encrypting each individual packet is significantly more than applying advanced authentication mechanism such as EAP-TLS standard (defined in 802.1x protocol and used at security layer 6 of these experiments).

The performance of TCP and UDP traffic types can also be compared. When changing from TCP (connection-oriented environment) to UDP (connection-less environment), the packet

\footnotetext{
${ }^{30}$ Refer to [3] (Section 3.3.1), [14] and [24] for information about WEP encryption standard.

${ }^{31}$ This indicates the infeasibility of using RC4 over 802.11 networks [14].
} 
drop rate increased by $81.3 \%$ on average. Under both traffic types, the network performance degraded as WEP encryption was applied (at security layers $4,5,7,8$, described in Section 3.2.3). Yet, the performance of TCP was significantly lower than UDP (the TCP throughput was $21.6 \%$ of that of UDP). This poor performance can be justified by the fact that TCP protocol applies congestion control mechanism, when the network is congested and the protocol detects packet drop. Figure 3-8 and 3-9 illustrate the performance of both traffic types.

As the number of clients increased, the overall throughput decreased, due to the effect of collisions and backoffs. The average throughput of the network decreased by $49.5 \%$ when the experiments were conducted using two clients and $66.5 \%$ when using three clients. Similar patterns were observed for both TCP and UDP traffic types, under all security layers.

Four different packet sizes $(100,500,1000$, and 1500) bytes were chosen in order to evaluate their impacts on the throughput of the congested wireless LAN under three different security mechanisms (MAC authentication, WEP encryption, and WEP encryption combined with $802.1 \mathrm{x}$ authentication). The packet size of 1000 bytes resulted in best throughput for both TCP and UDP traffic types under three security layers (see Section 3.4.3). The results confirmed those published in [41]. Due to the collision avoidance mechanism of the $802.11 \mathrm{~b}$ protocol, the traffic overheads introduced by the control frames RTS/CTS (see Section 2.2.4) and the ACK frames diminishes, as the packet gets larger. The packets were fragmented before they reach the MTU of 1500 bytes, so the performance of the network decreased, when fragmentation occurred.

\subsubsection{Limitations}

In general, there are several limitations associated with the experiments. Experiments were conducted in a confined environment, which may not simulate the real world. Real world factors such as environmental effects are important in wireless networks, since radio frequency transmission is influenced by other technologies operating in the same frequency band such as microwaves and weather conditions (see Section 2.3). These interferences may affect the performance results of a wireless network. Additionally, the experimental setup was confined to a single room. Factors such as wall interference degrade performance in actual wireless networks installed in a building. It was decided to run the experiments in the same room to eliminate as many environmental effects as possible.

One inherent limitation is using equipment containing vendor-specific implementations of packet handling, encryption algorithms and feature sets. Different vendors provide different capabilities, and as prior literature has shown [40], different hardware implementation can affect the performance.

The results determined from the effect of adding more clients on performance (Section 3.4.2) cannot be generalised to more than three clients, due to equipment limitation. However, the aim of this research was to evaluate the effect of security on the performance of congested networks. Experimenting with three clients was enough to create congestion, in order for the impact of security to be studied.

This research examined one type of 802.11 wireless networks, the infrastructure mode; the results might not be applicable to ad hoc wireless networks. Furthermore, the research focused on $802.11 \mathrm{~b}$ (as it is the most popular standard for Wireless LAN networking), and did not evaluate the security performance of other 802.11 standards such as $802.11 \mathrm{a}$ or $802.11 \mathrm{~g}$ (see Section 2.2.1). 


\section{Chapter 4}

\section{Future Work}

The experiments used EAP-TLS authentication, which uses digital certificates in order to authenticate the users. Protected EAP (PEAP) [38], proposed by RSA, Cisco and Microsoft, is another $802.1 \mathrm{x}$ mechanism. It provides mutual authentication and key generation, protects user authentication and supports quick re-authentication. Further research could be carried out to investigate the effect of PEAP on performance of congested and un-congested networks. Another research area that can be undertaken is to evaluate the effect of WPA (Wi-Fi Protected Access), which is an extension to 802.11 ESN (see Section 2.4.3), as well as software versus hardware implementations of encryption, on performance of wireless networks.

Roaming supports client stations moving freely from one cell (the AP coverage area) to another and requires the interaction between APs and Distribution Systems (see Figure 2-4). Users can move between the cells. When it occurs, transferring of credentials is necessary to ensure a secure connection. This scenario can involve reinitiating a search for an AP in the same way the client would, when it is initialised, or using other methods, such as referencing a table built during the previous association [47]. This research was limited to a single AP. Future research is recommended with multiple APs carrying out the experiments similar to the ones presented in this report, considering roaming factors. As mobility increases from wireless LANs to wireless WANs (seamless handoffs between the two), further research could be done to examine the ability of maintaining a secure connection without reassociation and re-authentication.

As outlined in the Section 3.5.1, this research examined one type of wireless network, the 802.11 standard; the results might not be applicable to all types of wireless networks. It also concentrated on the infrastructure mode of the 802.11 standards. This might prevent generalisation of the performance or security results to the ad-hoc mode of 802.11 wireless networks (see Section 2.2.3). Furthermore, the research focused on $802.11 \mathrm{~b}$ (Wi-Fi) protocol, as it is the most popular standard for wireless LAN networking. Evaluating the security performance of other 802.11 standards such as upcoming $802.11 \mathrm{~g}$, would be a foreseeable extension to this work. 


\section{Chapter 5}

\section{Conclusions}

This research was successful in investigating the performance and security issues of IEEE 802.11 wireless LANs with the layered security model, using multiple clients. It studied the interaction between different security layers and their effects on performance (response time and throughput) of congested and un-congested networks.

The ANOVA analysis confirmed that the security layers significantly differ from each other in their effects on the throughput of the network. When the network was not saturated (uncongested), the performance degraded as the quality of the security protection increased. In this situation, certificate-based authentication (EAP-TLS) increased response times and decreased throughput more than WEP encryption. However, when the network was congested, the performance was significantly low when WEP encryption was in place. In other words, WEP encryption affected the performance significantly more than certificate-based authentication. As the number of clients increased, the overall throughput decreased, due to the effect of collisions and backoffs.

The performance operating with TCP was significantly less than with UDP traffic, due to the congestion control mechanism, implemented by the TCP protocol. On the other hand, since UDP is a connectionless protocol, the rate of packet drop was more than with TCP traffic. Under both traffic types, the network performance degraded as WEP encryption was applied.

The research also evaluated the effect of different TCP and UDP packet sizes on network performance, when utilising different security mechanisms. It was concluded that the larger the packet size, the better the performance. Similar patterns were observed for MAC authentication, WEP encryption, and WEP encryption combined with $802.1 \mathrm{x}$ authentication.

Future work includes investigating the security performance of emerging IEEE 802.11 standards such as $802.11 \mathrm{~g}$ protocol using software and hardware implementations of encryption. Extending the security architectures to multiple APs and across wireless LAN/3G interfaces is also recommended. 


\section{Bibliography}

[1] Gast, M. (2002). Chapter 6: Security, Take 2: 802.1x, 802.11 Wireless Networks: The Definitive Guide. O'Reilly. ISBN 0-596-00183-5. April.

[2] Gast, M. (2002). Chapter 15: 802.11 Network Deployment, 802.11 Wireless Networks: The Definitive Guide. O'Reilly. ISBN 0-596-00183-5. April.

[3] Karygiannis, T., \& L. Owens. (2002). Draft: Wireless Network Security - 802.11, Bluetooth and Handheld Devices. USA. National Institute of Standards and Technology.

[4] Gast, M. (2002). Chapter 1: Introduction to Wireless Networks, 802.11 Wireless Networks: The Definitive Guide. O'Reilly. ISBN 0-596-00183-5. April.

[5] Wong, J. (2002). Performance Investigation of Secure 802.11 Wireless LANs: Raising the Security Bar to Which Level? University of Canterbury, Christchurch, NZ.

[6] Gast, M. (2002). Chapter 3: The 802.11 MAC, 802.11 Wireless Networks: The Definitive Guide. O'Reilly. ISBN 0-596-00183-5. April.

[7] Task Group i. (2002). TGi Security Overview, IEEE, Inc. Document number IEEE 802.11-02/114r1.

[8] Hannikainen, M., T. D. Damalainen, M. Niemi, \& J. Saarinen. (2002). Trends in Personal Wireless Data Communications. Computer Communications, 25. Elsevier. Page(s): 84-99.

[9] Gast, M. (2002). Chapter 2: Overview of 802.11 Networks, 802.11 Wireless Networks: The Definitive Guide. O'Reilly. ISBN 0-596-00183-5. April.

[10] Mishra, A., N. L. Petroni, \& B. D. Payne. (2003). Open1x -- Open Source Implementation of IEEE 802.1x. http://www.open1x.org/. June.

[11] Chen, J. C. (2001). Measured Performance of 5-GHz 802.11a Wireless LAN Systems. Atheros Communications, Inc. 27 August.

[12] Kapp, S. (2002). 802.11: leaving the wire behind. Internet Computing, IEEE. Volume: 6 Issue: 1. Page(s): $82-85$. February.

[13] Chevillat, P., \& W. Schott. (2001). Wireless Access Technology Beyond $3 G$. Paper presented at the Wireless World Research Forum (WWRF), Munich. 7 March. 
[14] Gast, M. (2002). Chapter 5: Wired Equivalent Privacy (WEP), 802.11 Wireless Networks: The Definitive Guide. O'Reilly. ISBN 0-596-00183-5. April.

[15] Fluhrer, S., I. Mantin, \& A. Shamir. (2001). Weaknesses in the Key Scheduling Algorithm of RC4. Eighth Annual Workshop on Selected Areas in Cryptography, August.

[16] Potter, B., \& B. Fleck. (2002). Chapter 1: A Wireless World, 802.11 Security. O’Reilly \& Associates. ISBN 0-596-00290-4. December.

[17] Stallings, W. (2001). IEEE 802.11: moving closer to practical wireless LANs. IT Professional. Volume: 3 Issue: 3. Page(s): 17 -23. June.

[18] IEEE Std. 802.11a (1999). Supplement to ANSI/IEEE. Std 802.11, 1999 Edition. Part 11: Wireless LAN Medium Access Control (MAC) and Physical Layer (PHY) Specifications: Higher Speed Physical Layer (PHY) in the $5 \mathrm{GHz}$ band. IEEE, Inc.

[19] IEEE Std. 802.11b (1999). Supplement to ANSI/IEEE. Std 802.11, 1999 Edition. Part 11: Wireless LAN Medium Access Control (MAC) and Physical Layer (PHY) Specifications: Higher Speed Physical Layer (PHY) Extension in the 2.4 GHz band. IEEE, Inc. ISBN 0-7381-1811-7. September.

[20] TGg. (2002). Task Group 802.11g, http://grouper.ieee.org/groups/802/11/ index.html

[21] Walker J. R. (2000). IEEE P802.11 Wireless LANs: Unsafe at any key size; An analysis of the WEP encapsulation. Document Number: IEEE 802.11-00/362. 27 October.

[22] Datanamics White paper. (2002). The Current State of Wireless LAN Technology. http://www.datanamicsinc.com/White\%20Papers/Wireless\%20LAN\%20Technology.pdf

[23] Geier, J. (2002). 802.11 Medium Access Methods. 802.11 Planet - The Source for Wi-Fi Business and Technology. http://www.80211-planet.com/tutorials. November.

[24] ANSI/IEEE. Std 802.11 (1999). Part 11: Wireless LAN Medium Access Control (MAC) and Physical Layer (PHY) Specifications. New York. First Edition. IEEE, Inc. ISBN 0-7381-1658-0. 20 August.

[25] Geier, J. (2002). 802.11 Beacons Revealed. 802.11 Planet - The Source for WiFi Business and Technology. http://www.80211-planet.com/tutorials. October. 
[26] Borisov, B., I. Goldberg, \& D. Wagner. (2001). Intercepting Mobile Communications: The Insecurity of 802.11. Seventh Annual International Conference on Mobile Computing and Networking. ACM. 16-21 July.

[27] Arbaugh, W. A., N. Shankar, \& Y. C. Wan. (2001). Your 802.11 Wireless Network Has No Clothes. University of Maryland. Maryland. 30 March.

[28] Housley, R., D. Whiting, \& N. Ferguson. (2002). IEEE P802.11 Wireless LANs: Alternate Temporal Key Hash. Document Number: IEEE 802.11-02/282r2. IEEE Task Group I. 23 April.

[29] TGi. (2002). Task Group 802.11i: IEEE, Inc. http://www.ieee802.org/11.

[30] Geier, J. (2002). Minimizing 802.11 Interference Issues. 802.11 Planet - The Source for Wi-Fi Business and Technology. http://www.80211-lanet.com/tutorials. January.

[31] Xiao, Y., \& J. Rosdahl. (2002). Throughput Limit for IEEE 802.11. IEEE 802.11 Working Group. May. Document Number: IEEE 802.11-02/291r0.

[32] IEEE Std. 802.1x (2001). Port-Based Network Access Control. New York. IEEE, Inc. ISBN 0-7381-2627-5. 25 October.

[33] InterLink Networks. (2002). Introduction to 802.1x for Wireless Local Area Networks. InterLink Networks.

http://www.interlinknetworks.com/images/resource/802_1X for_Wireless_LAN.pdf

[34] Potter, B., \& B. Fleck. (2002). Chapter 14: Authentication and Encryption, 802.11 Security. O’Reilly \& Associates. ISBN 0-596-00290-4. December.

[35] Congdon, P., B. Aboba, T. Moore, A. Smith, G. Zorn, \& J. Roese. (2002). IEEE 802.1x RADIUS Usage Guidelines. Internet-Draft. Internet Engineering Task Force. June.

[36] Aboba, B., \& D. Simon. (1999). PPP EAP TLS Authentication Protocol. RFC 2716. Internet Engineering Task Force. October.

[37] Dierks, T., \& C. Allen. (1999). The TLS Protocol Version 1.0, RFC 2246. Internet Engineering Task Force.

[38] Palekar A., D. Simon, G. Zorn, J. Salowey, H. Zhou, \& S. Josefsson (2003). Protected EAP Protocol (PEAP)v2. Internet Draft. Internet Engineering Task Force. 26 October.

[39] Bing B. (1999). Measured performance of the IEEE 802.11 wireless LAN. Local Computer Networks, 1999. LCN '99. Page(s): 34 -42, 18-20 October. 
[40] Vasan, A., \&. A. U. Shankar. An Empirical Characterization of Instantaneous Throughput in 802.11b WLANs. Department of Computer Science, University of Maryland. http://www.cs.umd.edu/ shankar/Papers/802-11b-profile-1.pdf

[41] Amaro, J., \& R. P. Lopes. (2001). Performance Analysis of a Wireless MAN. Network Computing and Applications. Page(s): 358-361, 8-10 October.

[42] Kamerman, A., \& G. Aben. (2000). Net throughput with IEEE 802.11 wireless LANs . Wireless Communications and Networking Conference, 2000. WCNC. 2000 IEEE, Volume: 2 ,Page(s): 747 -752 vol. 2. 23-28 September.

[43] Blunk, L., \& J. Vollbrecht. (1998). PPP Extensible Authentication Protocol (EAP), RFC2284: Internet Engineering Task Force.

[44] Xylomenos G., \& G.C. Polyzos (1999). TCP and UDP performance over a wireless LAN. INFOCOM '99. Eighteenth Annual Joint Conference of the IEEE Computer and Communications Societies. Proceedings. IEEE, Volume: 2 , 21 25. Page(s): 439 -446 vol.2. March.

[45] Williamson C. (2001). Internet Traffic Measurement. Department of Computer Science, University of Calgary. 24 November.

http://pages.cpsc.ucalgary.ca/ carey/papers/measurements.pdf

[46] Tourrilhes, J. (1998). Packet frame grouping: improving IP multimedia performance over CSMA/CA. Universal Personal Communications, 1998. ICUPC '98. IEEE 1998 International Conference, Volume: 2 , Page(s): 1345 1349 vol.2. 5-9 Oct.

[47] Convery, S., \& D. Miller. (2003). SAFE: Wireless LAN Security in Depth, version 2. White paper. Cisco Systems, Inc.

http://www.cisco.com/warp/public/cc/so/cuso/epso/sqfr/safwl_wp.pdf

[48] Chandran-Wadia L., S. Mahajan, \& S. Iyer. Throughput Performance of the Distributed and Point Coordination Functions of an IEEE 802.11 Wireless LAN, K. R. School of Information Technology, Department of Electrical Engineering, Indian Institute of Technology, Bombay. http://www.it.iitb.ac.in/ sri/papers/dot11-iccc02.pdf

[49] Yang, S. J. (2001). An Approach to Modelling Performance Evaluation on the Ethernet with QoS Parameters. International Journal of Network Management, 11. John Wiley \& Sons, Ltd. Page(s):91-101.

[50] Bradner, S., \& J. McQuaid. (1999). Benchmarking Methodology for Network Interconnect Devices. RFC 2544. Internet Engineering Task Force. March.

[51] Microsoft. (2002). Wireless 802.11 Security with Windows XP. Microsoft. http://www.microsoft.com//windowsxp/pro/techinfo/administration/wirelesssecurity/XP $\underline{80211 \text { Security.doc }}$ 
[52] Ikkurthy, P., \& M. A. Labrador. (2002). Characterization of MPEG-4 traffic over IEEE 802.11b wireless LANs. Local Computer Networks, 2002. Proceedings. LCN 27th Annual IEEE Conference, Page(s): 421 -427, 6-8 November.

[53] Proxim: Wi-Fi and Broadband Wireless Networking, ORiNOCO Classic Gold PC Card, http://www.proxim.com/learn/library/datasheets/gold_pccard.pdf

[54] Proxim: Wi-Fi and Broadband Wireless Networking, ORiNOCO AP-2000 http://www.proxim.com/learn/library/datasheets/AP-2000_US.pdf

[55] Bing, B., \& R. Subramanian. (1998). A Novel Technique for Quantitative Performance Evaluation of Wireless LANs. Computer Communications, 21. Elsevier. Page(s): 833-838.

[56] Held, G. (2001). The ABCs of IEEE 802.11. IT Professional, 3(6). IEEE, Inc. Page(s): 49-52. November.

[57] Cali, F., M. Conti, \& E. Gregori. (1998). IEEE 802.11 wireless LAN: Capacity analysis and protocol enhancement. In Proceedings of INFOCOM.

[58] Orinoco. (2002). Principles of 802.1x Security. ORiNOCO Technical Bulletin 048/B. Lucent. April.

[59] Geier, J. (2002). 802.11 MAC Layer Defined. 802.11 Planet - The Source for Wi-Fi Business and Technology. http://www.80211-planet.com/tutorials. June. 


\section{Appendix A}

\section{Captured Data}

\begin{tabular}{|c|c|c|c|c|}
\hline \multirow{2}{*}{$\begin{array}{l}\text { Security } \\
\text { Layer }\end{array}$} & \multicolumn{2}{|c|}{ TCP } & \multicolumn{2}{|c|}{ UDP } \\
\hline & $\begin{array}{l}\text { TCP Throughput } \\
\text { (Kbytes/sec) }\end{array}$ & $\begin{array}{c}\text { TCP Response Time } \\
\text { (sec) }\end{array}$ & $\begin{array}{l}\text { UDP Throughput } \\
\text { (Kbytes/sec) }\end{array}$ & $\begin{array}{l}\text { UDP Response Time } \\
\text { (sec) }\end{array}$ \\
\hline 1 & 440.35 & 78.28 & 535.52 & 64.44 \\
\hline 1 & 457.14 & 76.09 & 535.77 & 64.42 \\
\hline 1 & 448.87 & 77.42 & 535.85 & 64.41 \\
\hline 1 & 440.11 & 77.31 & 535.41 & 64.66 \\
\hline 1 & 451.13 & 77.02 & 535.93 & 64.48 \\
\hline 2 & 471.23 & 73.76 & 536.64 & 64.32 \\
\hline 2 & 476.35 & 72.94 & 534.24 & 64.60 \\
\hline 2 & 464.47 & 74.82 & 534.21 & 64.60 \\
\hline 2 & 472.11 & 73.33 & 535.32 & 64.81 \\
\hline 2 & 469.6 & 72.98 & 534.65 & 64.27 \\
\hline 3 & 482.69 & 72.0 & 541.24 & 63.76 \\
\hline 3 & 461.92 & 75.23 & 537.51 & 64.21 \\
\hline 3 & 450.72 & 77.09 & 530.42 & 65.30 \\
\hline 3 & 461.76 & 75.82 & 535.12 & 64.80 \\
\hline 3 & 465.14 & 76.3 & 538.34 & 65.14 \\
\hline 4 & 66.57 & 524.63 & 245.77 & 134.05 \\
\hline 4 & 70.29 & 496.64 & 245.09 & 134.25 \\
\hline 4 & 65.88 & 530.07 & 245.99 & 133.94 \\
\hline 4 & 67.4 & 522.07 & 245.15 & 133.98 \\
\hline 4 & 66.33 & 530.44 & 245.33 & 134.15 \\
\hline 5 & 66.40 & 525.41 & 243.12 & 135.56 \\
\hline 5 & 61.15 & 571.37 & 248.2 & 133.13 \\
\hline 5 & 64.15 & 544.2 & 244.53 & 134.69 \\
\hline 5 & 63.12 & 563.2 & 245.23 & 133.87 \\
\hline 5 & 63.87 & 529.77 & 246.97 & 134.64 \\
\hline 6 & 454.8 & 76.41 & 533.25 & 64.71 \\
\hline 6 & 453.99 & 76.54 & 543.23 & 63.53 \\
\hline 6 & 454.44 & 76.48 & 543.92 & 63.45 \\
\hline 6 & 454.98 & 76.45 & 543.11 & 63.48 \\
\hline 6 & 455.03 & 76.54 & 533.77 & 63.97 \\
\hline 7 & 67.45 & 517.82 & 244.25 & 134.97 \\
\hline 7 & 64.51 & 540.7 & 245.35 & 134.37 \\
\hline 7 & 61.45 & 568.39 & 243.76 & 135.33 \\
\hline 7 & 63.32 & 560.17 & 244.87 & 134.45 \\
\hline 7 & 64.77 & 562.12 & 243.44 & 134.49 \\
\hline 8 & 64.79 & 559.54 & 251.16 & 128.82 \\
\hline 8 & 60.90 & 573.73 & 242.76 & 131.05 \\
\hline 8 & 59.86 & 583.25 & 244.59 & 134.50 \\
\hline 8 & 61.76 & 570.15 & 246.59 & 132.50 \\
\hline 8 & 60.34 & 581.46 & 244.03 & 131.70 \\
\hline
\end{tabular}




\section{Appendix B}

\section{Configuration Procedure}

The following describes the modification made to the wireless LAN architecture used in [5] in order to enhance it to support multiple clients. Section 1 presents the enhancements made to the clients side and Section 2 illustrates the modifications made to the server side.

\section{B.1 The Clients side}

After installing ORiNOCO Wireless LAN Gold Cards ${ }^{32}$ on Windows XP client machines, the client machines were assigned suitable IP addresses and subnet masks. The IP address of gateway and DNS server on each machine was also specified (See Figure B-1).

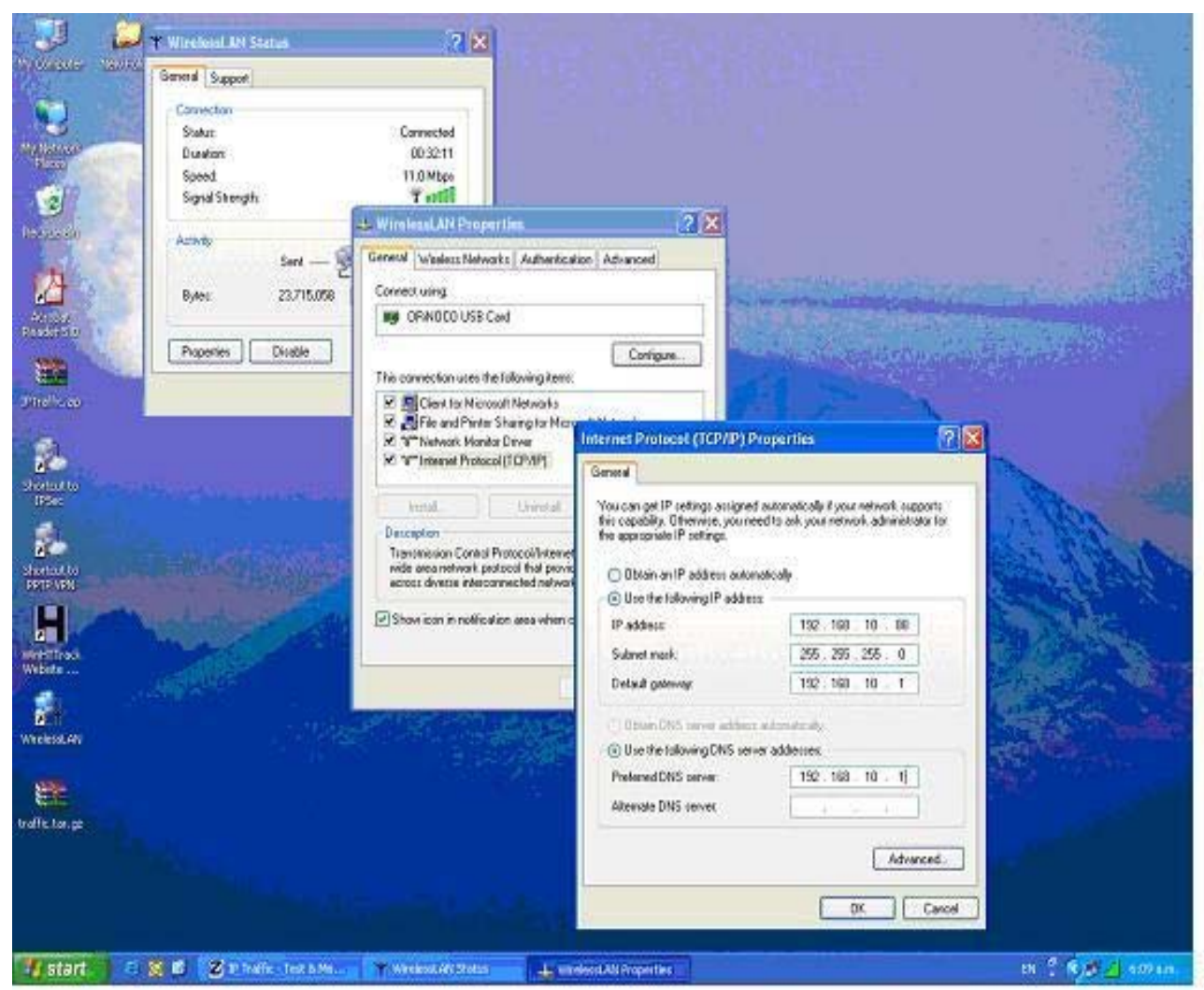

Figure B-1: Specifying the IP addresses

The domain and computer name for each client was specified, as shown in Figure B-2.

\footnotetext{
${ }^{32}$ Donated by Lucent (www.lucent.com)
} 


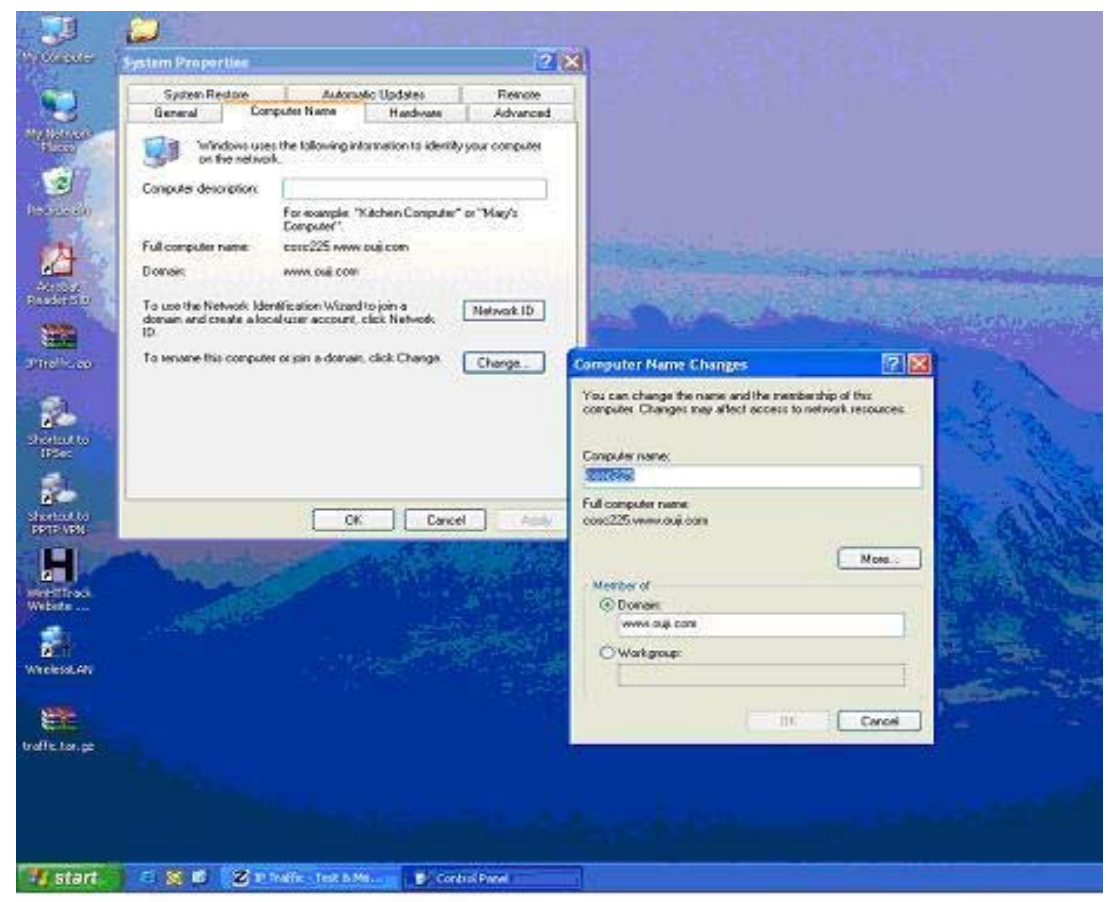

Figure B-2: Configuration of computer and domain name

Figure B-3 illustrates the specification of WEP authentication and encryption, using 45-bit key length. WEP encryption was implemented at security layers 4, 5, 7 and 8 .

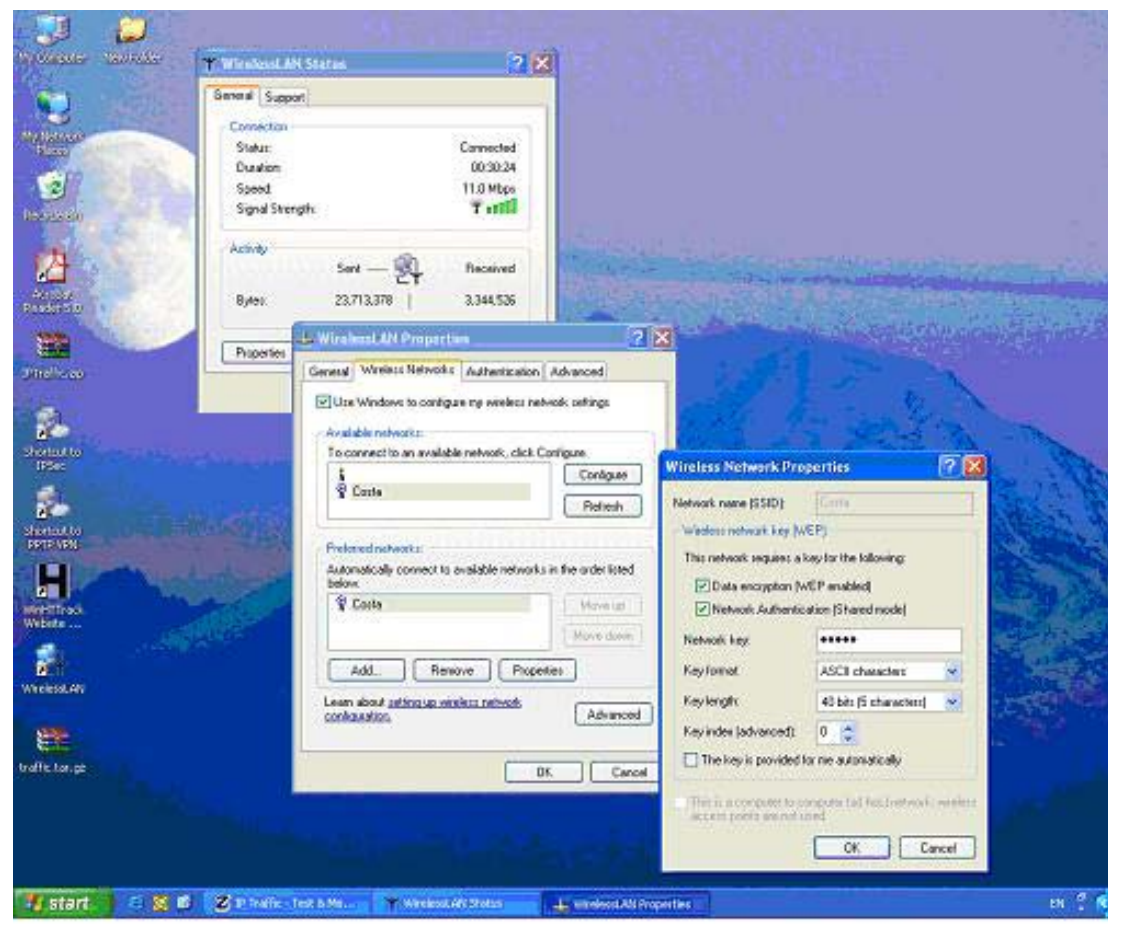

Figure B-3: Configuration of WEP encryption

The IEEE 802.1x authentication protocol (EAP-TLS) was implemented at security layers 6, 7 and 8. Figure B-4 illustrates the process of specifying the properties for each digital certificate. 
The certificates were issued by CALAB, the Certification Authority, which was set up on server machine (See Figure B-12). The certificates were used for authenticating the users.

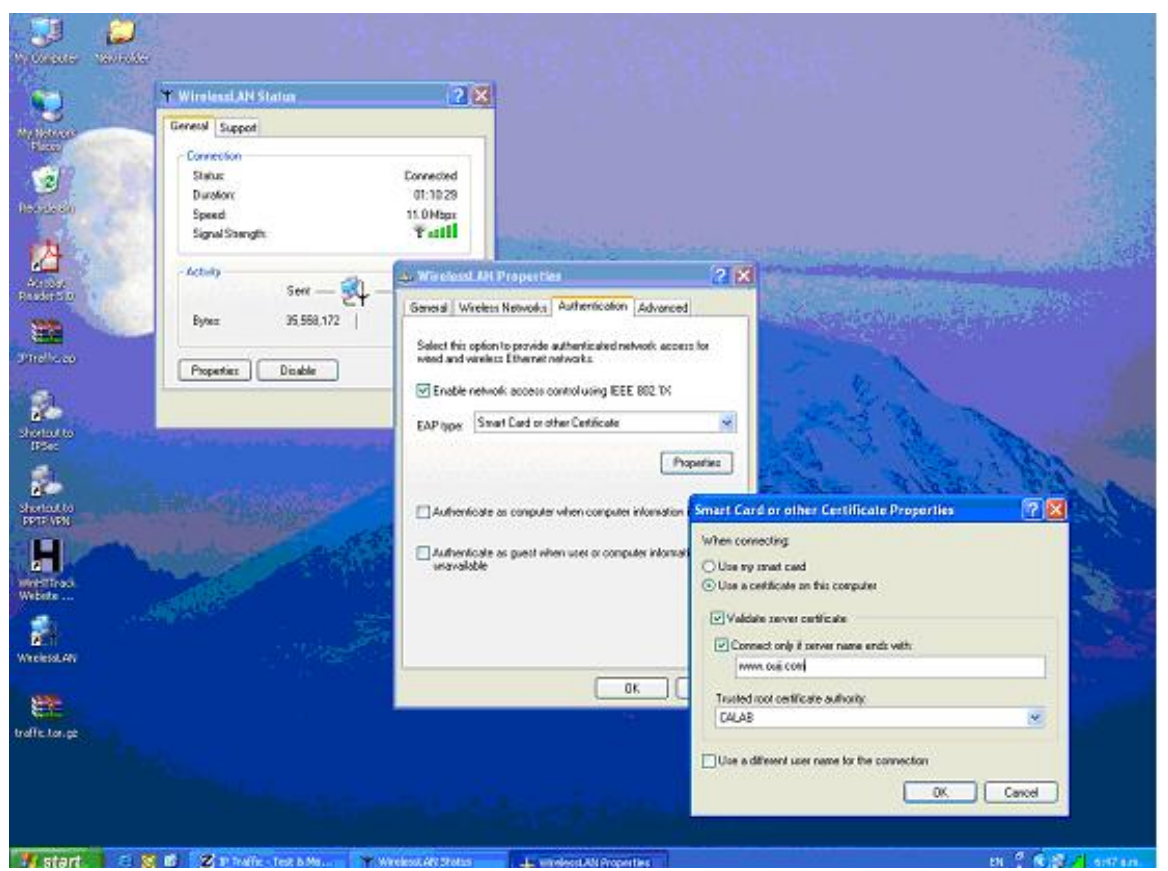

Figure B-4: Specifying the certificate properties

\section{IP Traffic}

IP Traffic, the traffic generator used in this research, has two operating modes. Figure B-5 shows the client mode.

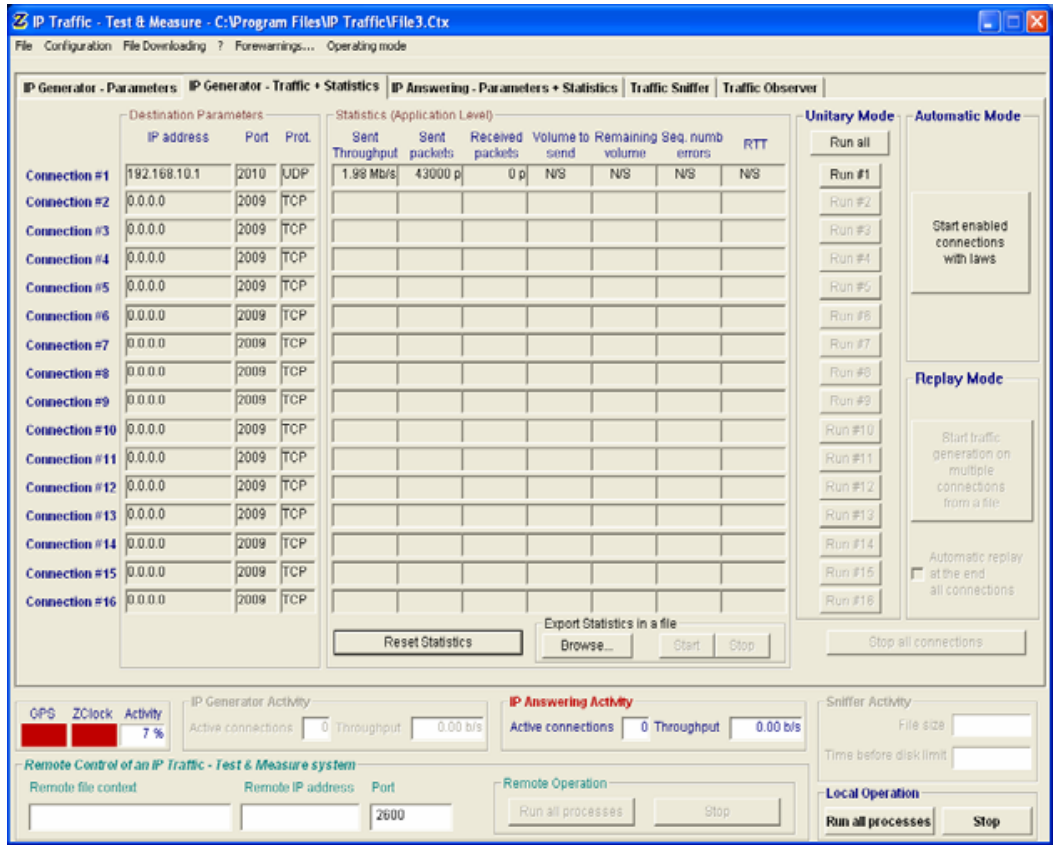

Figure B-5: IP Traffic on Clients side 
As described in Section 3.2.5 of the report, one active connection was used from each client machine to send and receive traffic. The IP address of the server and type of traffic was also specified.

\section{B.2 The Server Side}

\section{Access Point configuration}

Figure B-6 illustrates the MAC authentication tab on the AP. The IP addresses of new client machines were added to MAC Access Control Table. The MAC authentication security was in place from security layer 2 onwards.

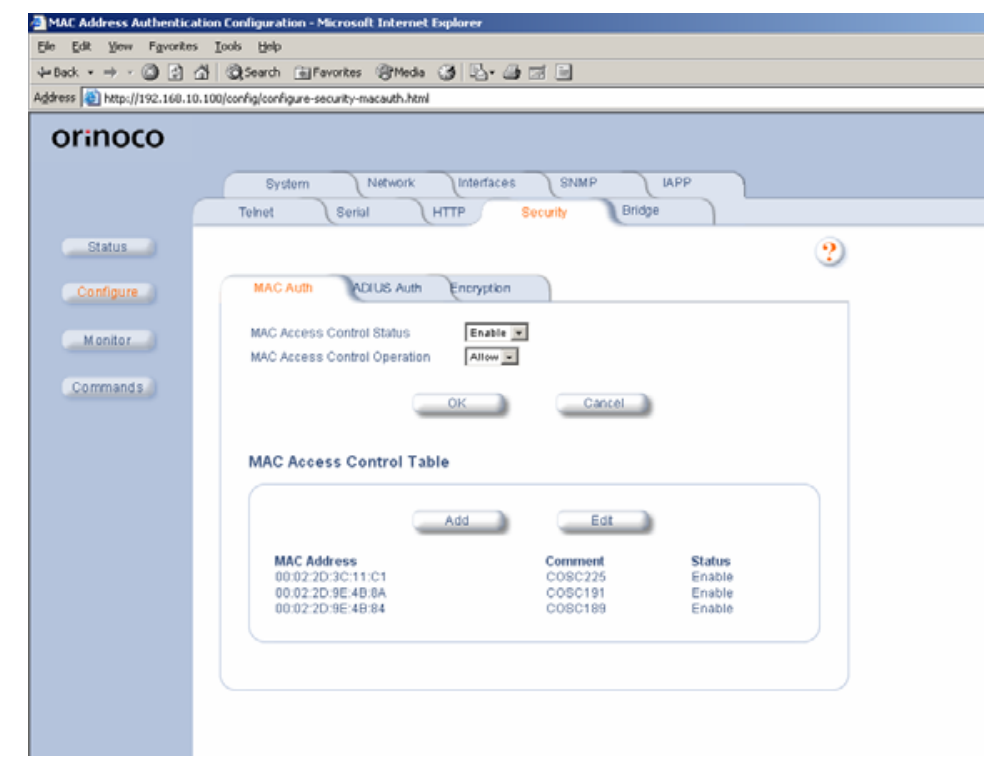

Figure B-6: Implementing the MAC authentication

Figure B-7 shows the specification of RADIUS authentication on the AP. RADIUS authentication was activated on AP at security layers 6,7 and 8 .

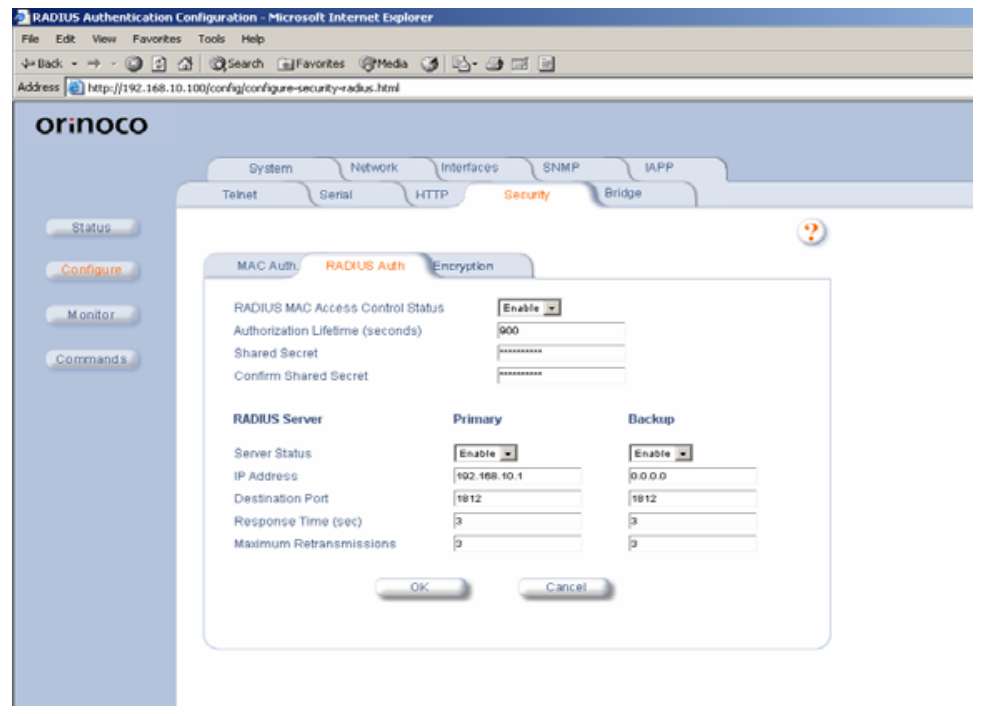

Figure B-7: Activating the Radius Server on the AP 
The IEEE $802.1 x$ security was used in security layers 6,7 and 8 . Figure B-8 illustrates the encryption key parameters specified on the AP. These parameters were the same as the ones specified on the clients side (See Figure B-3).

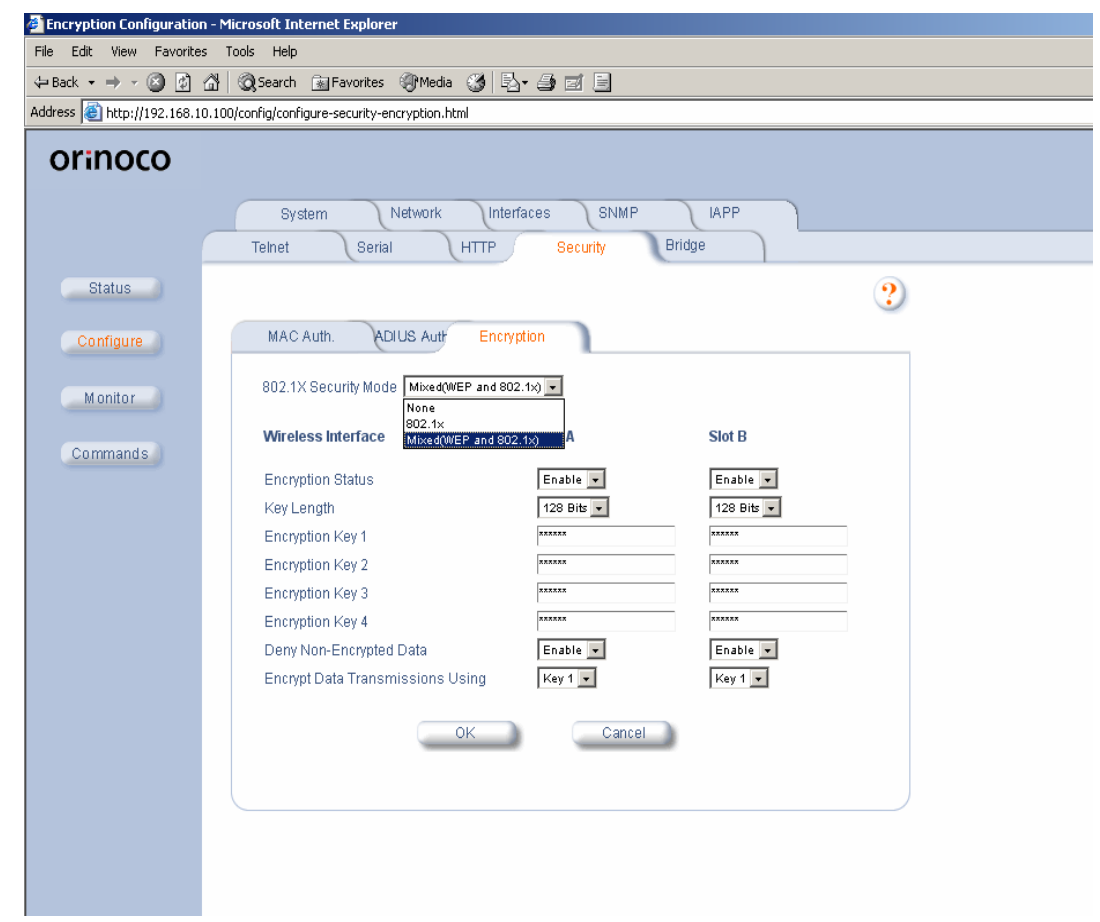

Figure B-8: 802.1x security model on the AP

\section{Server configuration}

The new client machines and new users were added to the Active Directory (AD), as shown in Figure B-9 and B-10.

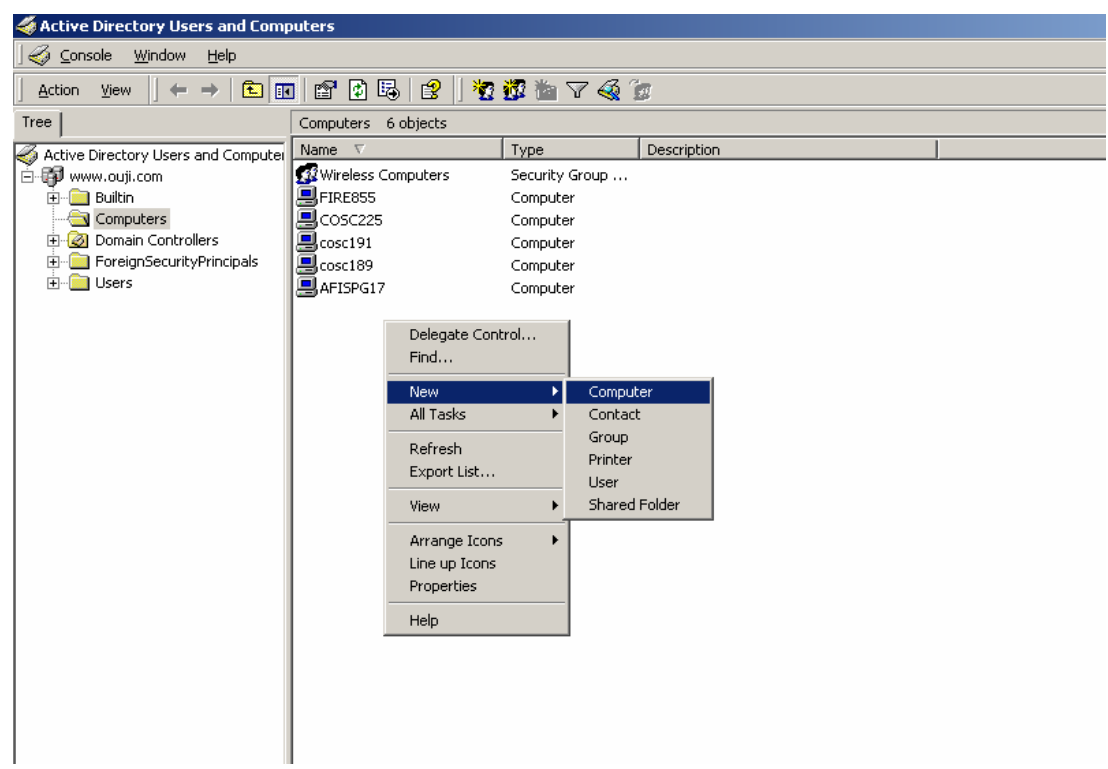

Figure B-9: Adding new machines to $A D$ 


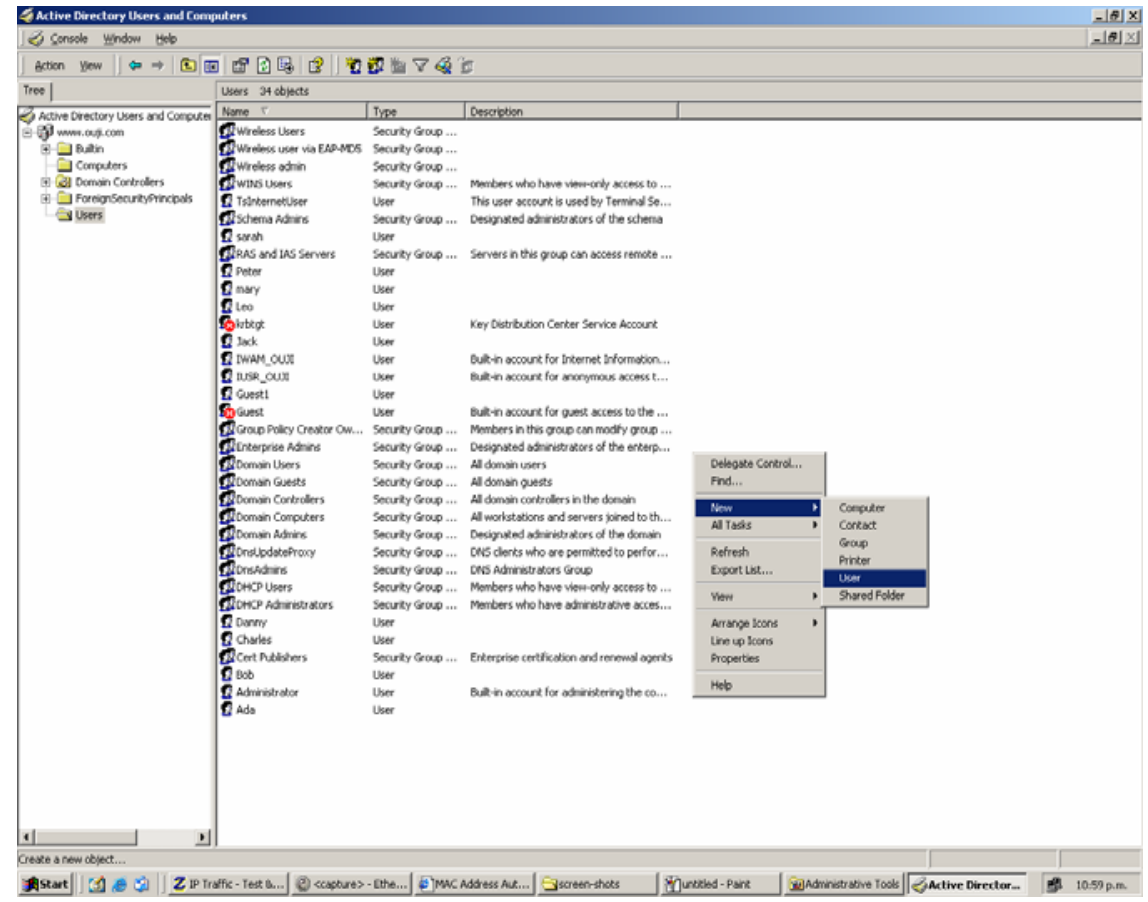

Figure B-10: Adding new users to $A D$

In the PKI configuration, the option for issuing automatic machine/computer certificates was enabled, as shown in Figure B-11.

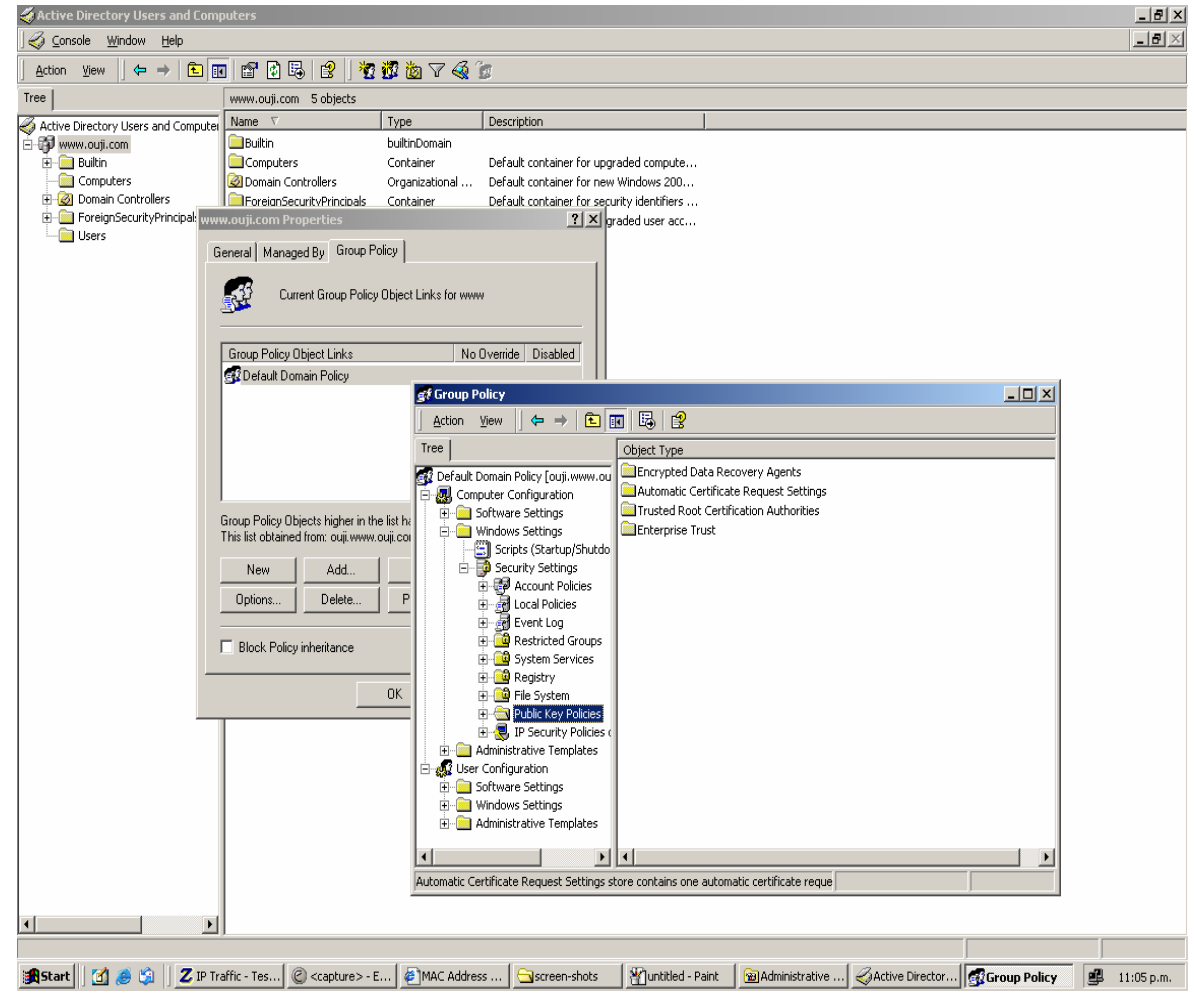

Figure B-11: Automatic issuing of computer certificate 
CALAB, the Microsoft certification authority, was used on the server side to issue certificate to the users. Figure B-12 illustrates the parameters specified during the process of issuing certificates. The certificates were stored on the server and were transferred to the client machines. In doing that, it was important to mark the keys as exportable ${ }^{33}$.

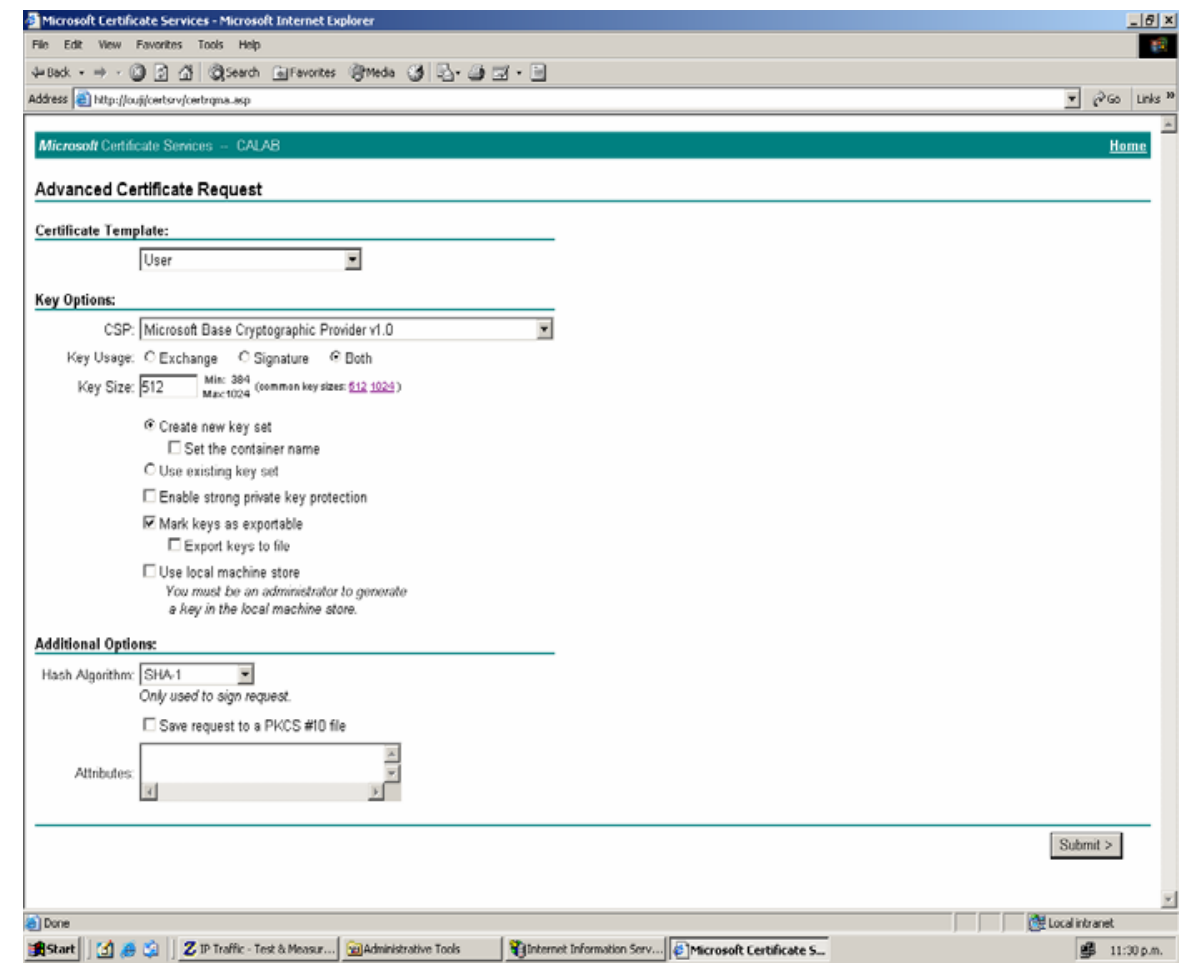

Figure B-12: Issuing certificates to network users

Figure B-13 illustrates the process of specifying the security mechanism on the server side (Remote Access Policy).

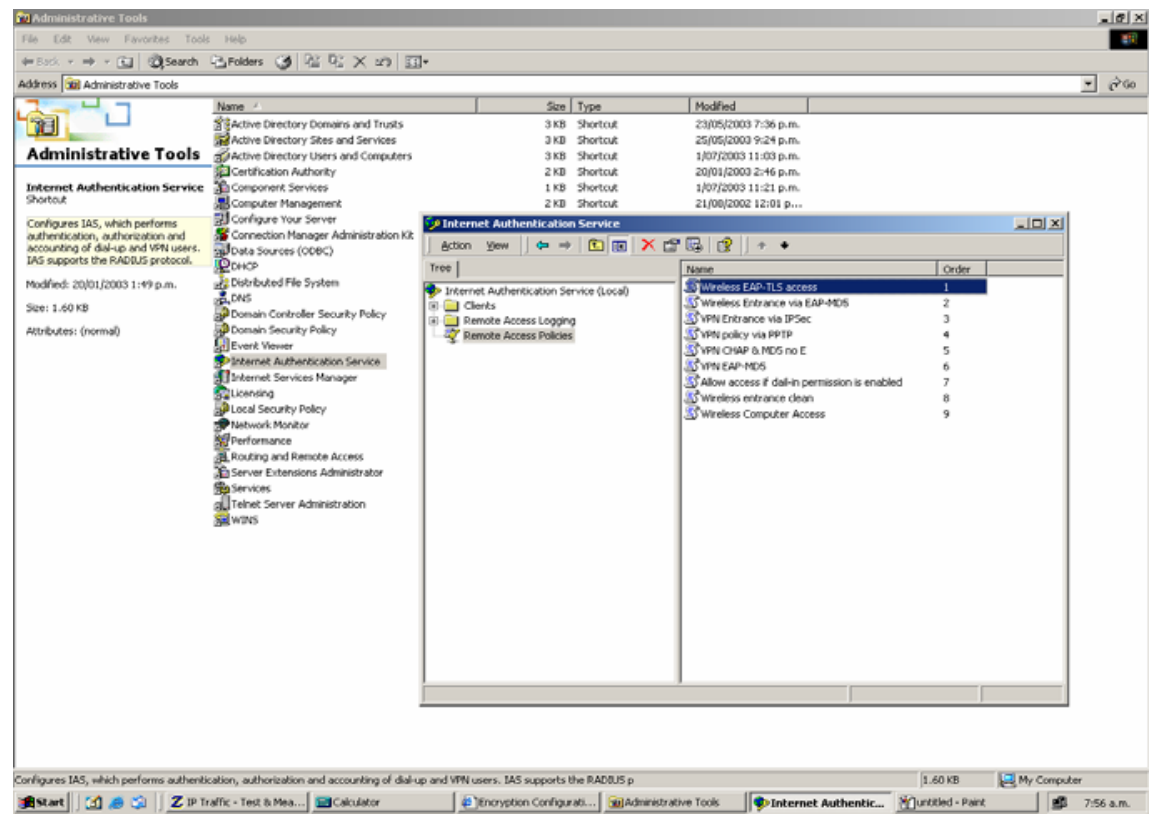

Figure B-13: Configuring the security mechanisms on server

${ }^{33}$ In order to access the certification authority on the server, the $\lfloor$ servernamelcertsrv address was used. 


\section{Ethereal}

As described in section 3.2.2 of the report, Ethereal was used to capture the traffic generated by IP Traffic tool between the server and the clients.

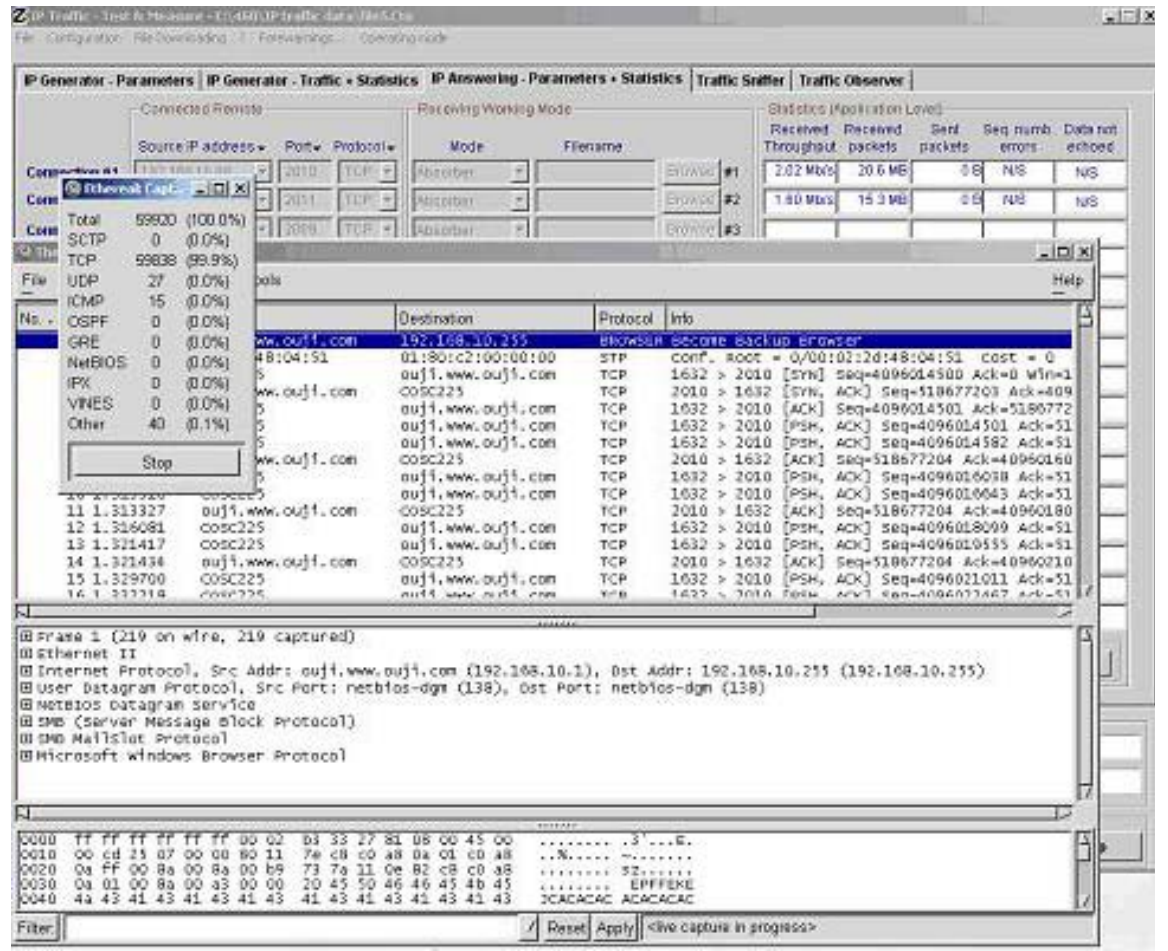

Figure B-14: Ethereal capturing TCP packets on server side

Figure B-14 illustrates the process of capturing TCP packets. The packets were then filtered and protocol statistics were used in order to calculate the throughout and response times of the network. 